\title{
Power Efficient Topology Control for Static Wireless Networks with Switched Beam Directional Antennas *
}

\author{
Vinod Namboodiri, Lixin Gao*, Ramakrishna Janaswamy \\ Department of Electrical and Computer Engineering, \\ University of Massachusetts at Amherst, Amherst, Massachusetts, 01003, USA
}

\begin{abstract}
Topology control problems are associated with assignment of power levels to nodes of a wireless network so that the resulting graph topology satisfies certain properties. In this paper we consider the problem of power-efficient topology control with switched beam directional antennas taking into account their non-uniform radiation pattern within the beamwidth. Previous work in the area have all assumed a uniform gain model with these antennas which renders antenna orientation insignificant as a parameter in topology control algorithms. We present algorithms that take into account a model of non-uniform gain with the objectives of minimizing the total power and maximum power to keep the network connected. We consider two cases: one where the antenna orientation is assumed given and another where the antenna orientation needs to be derived as well. For the first case, we present optimal and approximation algorithms for constructing power-efficient topologies. For the second case, we prove the problem to be NP-complete and present heuristic solutions along with approximation bounds. Through comparison of the two cases by simulation, significant reductions are shown in the maximum as well as total power required to keep the network connected for the second case, thus demonstrating the benefits of using antenna orientation as parameter in topology construction.
\end{abstract}

Key words: Wireless ad hoc network, Topology control algorithms, Directional antennas

ऋ A preliminary version of this work appeared in Proceedings of IEEE Mobile Ad Hoc and Sensor Systems, 2005 [1].

* Corresponding author.

Email addresses: vnambood@ ecs.umass.edu (Vinod Namboodiri), lgao@

ecs.umass.edu (Lixin Gao), janaswamy@ ecs.umass.edu (Ramakrishna

Janaswamy).

Preprint submitted to Elsevier Science 28 December 2006 


\section{Introduction}

A widespread approach to topology control with omni-directional antennas is to find a power assignment for all nodes such that cost metrics like throughput or power consumption are optimized subject to constraints such as network connectivity [2-7]. Such approaches to construct power-efficient topologies for wireless networks with omni-directional antennas have recently been adapted to the case of directional antennas [8-12]. Directional Antennas have received considerable attention the last few years due to the spatial-reuse they provide which increases the potential capacity of wireless ad-hoc networks $[13,14]$. Moreover, there are also benefits of power efficiency with directional antennas as they radiate power in only the desired directions. The gains of these antennas are typically much higher than the omni-directional case making them influential in reducing the power required between a transmitter and receiver. Two types of directional antennas have mainly been considered; steered beam and switched beam from the class of smart antennas. Steered beam directional antennas radiate power in only a certain direction, but the point of maximum gain can be steered as desired to always utilize the peak gain for transmitting and receiving. So the positioning of antennas, or antenna orientation, of nodes when deployed does not matter. However, these antennas require complex hardware and the cost makes them infeasible for most wireless ad-hoc networks. Switched beam directional antennas are less complex and cheaper, and thus, more feasible for ad-hoc wireless network settings in the coming few years [15]. However, the point of maximum gain cannot be steered by the communication system and is predefined based on the antenna orientation. Thus, if nodes are not communicating through the axis of maximum gain of each other, it could lead to considerably more power being required for that link.

The above mentioned work which focus on switched beam directional antennas ignore the effect of non-uniform gain and assume a constant uniform gain within the beamwidth [8-10,12]. The circular disk model used for omnidirectional antenna radiation pattern was simply cut into pie sections to model directional antennas. The reality is that the radiation pattern is far from uniform. We need to model the switched beam directional antenna radiation pattern with a radical approach that reflects the non-uniform gain within the beamwidth. For any algorithm, the challenge is to build topologies which use regions of higher gain as much as possible to reduce the power consumption. With uniform gain assumptions, the power required for communication between two nodes mainly depends only on the distance between them. But with a non-uniform radiation pattern, there is a new challenge of the gain in the desired direction also being a significant factor in deciding the required power. This requires that the antenna orientation of nodes be considered. The positioning of a node's antenna decides what power each of its communication links incur. Thus, topology control algorithms need to find power assignments 
as well as antenna orientation for each node to optimize the power-based cost metric under consideration.

In this paper, we address the issue of power-efficient topology control in a static wireless ad-hoc network setting with switched beam directional antennas, taking into account their non-uniform radiation pattern. We describe two different modes of communication possible with directional antennas and present a model which reflects the non-uniform radiation pattern of these antennas. We look at the problem of power-efficient topology construction along two dimensions: one, to minimize the maximum power used by any node of the network and two, to minimize the total power used by the nodes of the network. Further, we look at two circumstances where a topology control algorithm may be employed to find the most power-efficient topology (i) A network exists with all nodes already having fixed antenna orientations and the task is to come up with a power-efficient topology using the given antenna orientations of nodes. For the problem of minimizing the maximum power of a network, we present optimal centralized algorithms that build topologies using the non-uniform model of gain. We also provide approximation algorithms for minimizing the total power of the network. (ii) Before deployment, the topology control algorithm constructs a power-efficient topology using antenna orientations also as a parameter. On deployment, the derived antenna orientations and power levels of nodes are adjusted accordingly. We prove that finding an optimal power assignment for this scenario is NP-Complete. We provide heuristic algorithms for this scenario that construct topologies minimizing the maximum power and total power of the network. Moreover, approximation bounds are provided for these algorithms.

We evaluate our algorithms and find that using antenna orientation as a parameter in topology construction provides significant reduction in the maximum power and total power of the network. This reduction in power would not have been obtained by the existing practice of using a uniform model of gain, as then the antenna orientation would never have been considered a parameter in the first place.

The remainder of the paper is structured as follows. We introduce some concepts about directional antennas in Section II including the propagation model, two modes of communication and switched beam antenna model for non-uniform gain. Section III formally defines the problem statement and metrics we consider in this work. In Sections IV, and V we present algorithms to construct power efficient topologies for minimizing the maximum power as well as total power of the network. In Section VI we improve on the approximation bounds of our heuristic algorithms. We show through evaluation in Section VII that making the antenna orientation a parameter in topology construction results in significant reductions in maximum as well as total power of the network. Finally, Section VIII presents concluding remarks. 


\section{Directional Antennas}

In this section we introduce some related terminology about directional antennas. We also present our models and related assumptions before we formulate the problem in the following section.

\subsection{Gain and Beamwidth}

An omni-directional antenna radiates or receives energy equally well in all directions while a directional antenna transmits/receives more energy in one direction compared to others. The term gain is used to quantify the directionality of the antenna. The gain of an omni-directional antenna is typically taken as unity ${ }^{1}$.

Beamwidth is the angle subtended between the two points on either side of the direction of peak gain that are $3 \mathrm{~dB}$ down in gain. In terms of absolute values, a $3 \mathrm{~dB}$ drop in gain halves the peak gain. The more directional an antenna, smaller the beamwidth and higher the gain. However, due to presence of side lobes, two antennas with equal gain may not have the same beamwidth and vice-versa.

\subsection{Propagation Model}

The required transmit power for communication between a transmitter-receiver link is inversely proportional to the product of their gains and can be specified as

$$
P_{t}=\frac{c \cdot d^{\gamma}}{G_{t} \cdot G_{r}}
$$

based on the two-ray ground propagation model [16], where $P_{t}$ is the transmit power, $d$ is the distance between sender and receiver, $\gamma$ is the path loss exponent, $c$ a constant depending on factors like antenna height and $G_{t}$ and $G_{r}$ being the gain of transmitting and receiving antenna towards each other. For Omni-Directional antennas, $G_{t}$ and $G_{r}$ are unity. Hence power required is directly proportional to $d^{\gamma}$. With directional antennas, however, the gains have a role to play in deciding power required for communication, as described subsequently. It is assumed that the threshold power level above which a packet is received successfully is uniform for radios of all nodes.

$\overline{1}$ It is usually measured in decibels $(\mathrm{dB})$ and taken as $0 \mathrm{~dB}$, which equals unity in absolute terms 


\subsection{Mode of Communication}

The mode of communication with directional antennas can be classified into Directional-Omni (DO), or Directional-Directional (DD) based on the type of antenna used for transmission and reception across a communication link. DO links require more power than DD links as only the transmitter uses the higher gain providing directional antenna. However, coordination between the communicating nodes is easier. Due to omni-directional reception, now the sender can transmit packets directionally without worrying how the receiver's antenna is oriented. Omni-directional transmission with directional reception (OD) links are also an option, but suffer from requiring more power than DD links with no ease of coordination like DO links. Hence OD links are not considered in our work. This mode though is worth considering when the Effective Isotropic Radiated Power (EIRP) limits are a factor as is done in [17].

\subsection{Switched Beam Antenna Model}

We assume each node is capable of working in omni-directional mode too, either by adjusting the radiation pattern or having another omni-directional antenna. We do not consider varying the gain of the antennas for any other purpose and assume a constant beamwidth of $\theta$ for the directional mode. We use a simplifying assumption that the effects due to side-lobe interference are negligible. Furthermore we consider switched beam antennas with no nulls in between beams facing any direction. That is, there is $360^{\circ}$ coverage (this also implies that there is some overlap among the beams which we ignore for simplicity as shown in Figure 1). By antenna orientation we mean the angle between the first beam anticlockwise from the positive $\mathrm{x}$-axis and the $\mathrm{x}$-axis. The orientation of antenna in Figure 1 is $\Phi$. We will be concerned only with the azimuth plane for antenna orientation and radiation pattern in this work.

\subsubsection{Bounds on required power due to non-uniform gain}

Each beam's gain varies from a peak of $G_{\max }$ to $G_{\min }\left(G_{\min }=G_{\max } / 2\right)$ at its borders ${ }^{2}$. Thus, regardless of direction of transmission or reception, transmitter and receiver nodes will have at least half the maximum gain towards each other.

Thus, for DD links we have

$$
G_{\max } / 2 \leq G_{t}, G_{r} \leq G_{\max }
$$

$\overline{2 G_{\min }}=G_{\max } / 2$ follows naturally from the definition of beamwidth which is defined as angle between point of half gain on both sides of the axis of peak gain 


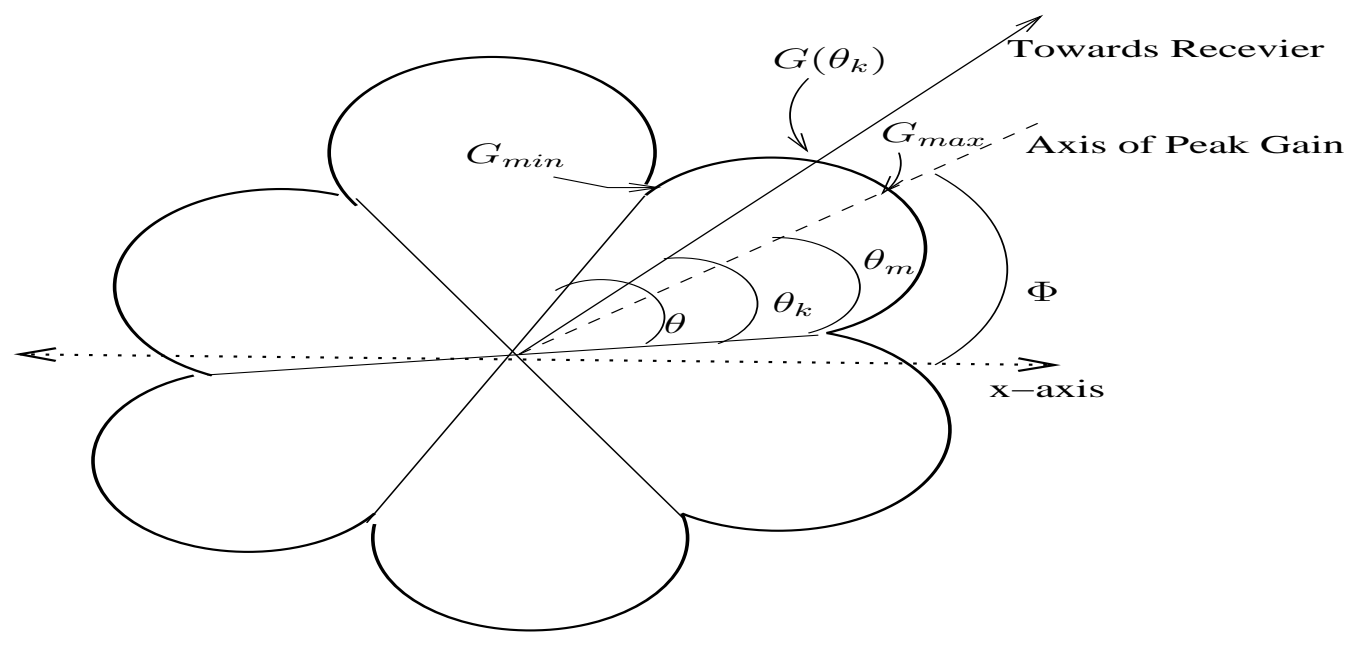

Fig. 1. Calculation of $G\left(\theta_{k}\right)$

or

$$
\frac{G_{\max }^{2}}{4} \leq G_{t} \cdot G_{r} \leq G_{\max }^{2}
$$

From Equation 1,

$$
\frac{4 . c . d^{\gamma}}{G_{\max }{ }^{2}} \geq P_{t} \geq \frac{c . d^{\gamma}}{G_{\max }^{2}}
$$

Similarly for DO links with $G_{r}$ unity, we have

$$
\frac{2 . c . d^{\gamma}}{G_{\max }^{2}} \geq P_{t} \geq \frac{c . d^{\gamma}}{G_{\max }^{2}}
$$

For simplicity, we take $c=1$. The important message here is that it is worth the effort to model non-uniform gain within the beamwidth. If we had assumed uniform gain to build our topologies we wouldn't have cared about the gain of communicating end nodes towards each other and, which as shown in Equations 4 and 5, makes a big difference in the power required for communication.

\subsubsection{Calculation of Gain}

Since our intention is to model the radiation pattern of a switched beam antenna with non-uniform gain within the beamwidth, we need a function to calculate the gain associated with communication in different directions. The gain from a transmitter towards a receiver is a function of the angle of the straight line joining the two nodes and the beam margin. That is, in Figure 1, $G\left(\theta_{k}\right)$ is the gain of transmitting antenna towards a receiver $k$ which is at an angle $\theta_{k}$. Also suppose $\theta$ and $\theta_{m}$ are the beamwidth and the half-beamwidth 
respectively. Then $G\left(\theta_{k}\right)$ can be modeled by the following Gaussian function:

$$
G\left(\theta_{k}\right)=G_{\max } \cdot e^{-\frac{\left(\theta_{k}-\theta_{m}\right)^{2}}{2 \sigma^{2}}}
$$

Here $\sigma$ can be calculated by using the known value of $G\left(\theta_{k}\right)$ at $\theta_{k}=\theta$.

$$
\begin{gathered}
G(\theta)=\frac{G_{\max }}{2}=G_{\max } \cdot e^{-\frac{\left(\theta-\theta_{m}\right)^{2}}{2 \sigma^{2}}} \\
\Longrightarrow \sigma=\frac{\theta-\theta_{m}}{\sqrt{2 \ln 2}}=\frac{\theta_{m}}{\sqrt{2 \ln 2}}
\end{gathered}
$$

The last equality in Equation 7 comes from the fact that $\theta=2 . \theta_{m}$. Thus, using Equation 6 along with Equation 7, each node can calculate the gain towards any direction. Combining this with Equation 1, the power required to reach another node can be calculated. More details about the above gaussian model can be found in [18].

\section{Problem Formulation}

In this section we describe the power metrics that we seek to optimize for, and formally define the optimization problems we will consider along with the connectivity requirements to be satisfied by our algorithms.

\subsection{Power Metrics}

Power efficiency for wireless networks has been measured primarily through two metrics; maximum power and total power. The latter is a more traditional metric trying to minimize the total power consumption in the wireless network. Energy is viewed as a network resource, with seemingly an endless supply at each node, costs being the only constraint. Maximum power used by any node, however, is a metric borne out of the newer applications of wireless networks where they may be operating unmanned until they die out. In such networks, energy is an individual resource. This metric supports the line of thought that a network's lifetime is maximized by trying to minimize each node's power consumption. Minimizing the total power consumption of the network might conserve some extra energy at some nodes, but this energy conserved at the expense of network disconnection may be of little value. Thus, based on the type of deployment and energy supply, both metrics have their own significance and the algorithms we present satisfy both these metrics. Let $C \in\{\operatorname{Max} P$, TotalP $\}$ represent the set of metrics we want to satisfy. 
Note that topology control algorithms can build truly energy-efficient networks only if the traffic pattern information in the network is also known. However, such algorithms would then need to dynamically adjust the topology to changing traffic patterns in the network. This is a difficult problem and an open area of research. Existing topology control algorithms have focused on a stand-alone approach where the nodes are assigned power levels to minimize a cost metric while keeping the network connected [7]. They do not take traffic patterns into consideration which may or may not be available to the algorithm. We will take this approach as well, primarily because our main focus is on demonstrating the benefits of using a non-uniform model of gain with switched beam directional antennas. This work can be extended to emerging algorithms that take into account knowledge of traffic patterns as well.

\subsection{Problem Definitions}

Definition 1 Power Assignment with Given Antenna Orientation (PAGA). Given a set $M=(N, L, \Phi)$, with a set of $\mathrm{B}$ beams of beamwidth $\theta$ per node $\in N$, and path loss function $\gamma$, find a per node minimal assignment of power $p: N \rightarrow Z^{+}$such that the induced graph is connected and satisfies $C$.

Definition 2 Power Assignment with Derived Antenna Orientation (PADA). Given a set $M=(N, L)$, with a set of B beams of beamwidth $\theta$ per node $\in N$, and path loss function $\gamma$, find a antenna orientation assignment $\Phi: N \rightarrow$ $\Phi, 0 \leq \Phi \leq \theta$ and a per node minimal assignment of power $p: N \rightarrow Z^{+}$such that the induced graph is connected and satisfies $C$.

The PAGA problem corresponds to settings where the antenna orientation is assumed given and not a parameter in topology construction. The solution to the PAGA problem would be useful in settings where each node's antenna orientation cannot be changed after deployment. That is, nodes find power assignments for themselves based on their fixed antenna orientation. This can be achieved either by sending information to an elected leader who executes the algorithm and returns everyone's power assignment or all nodes distributively execute the algorithm. This problem, however, may not be relevant in settings where a centralized authority could control the antenna orientation at the deployment stage. Previous work assumed uniform gain within the beamwidth to build topologies and thus did not use antenna orientation as a parameter. Hence, we use the solution to the PAGA problem also as a base case for comparison against the case where antenna orientation is a parameter in topology construction.

The PADA problem on the other hand deals with scenarios where the antenna orientations of each node can be calculated offline and deployed by a 
centralized authority or controlled by the node's user after deployment at the direction of an elected leader in the network. It is important to keep in mind that the communication system of a switched beam antenna cannot control the antenna orientation and needs external assistance.

\subsection{Connectivity Requirements}

We impose weak symmetry constraints ${ }^{3}$ on the communication graph.

Definition 3 Let $p$ be a power assignment for the set of nodes $N$ and let $G$ be the corresponding (directed) communication graph. The symmetric subgraph of $G$, denoted by $G_{S}$ is the undirected graph obtained from $G$ by removing unidirectional links. The power assignment problem with weak symmetry constraints then is to find a $G_{S}$ that is connected of minimum cost.

Symmetric links are a requirement for most routing and MAC protocols. For example, in the 802.11 MAC protocol the node that has a packet to send transmits a Request to Send (RTS) and awaits a Clear to Send (CTS) scheme from the next hop receiver. If the link were not symmetric, either the RTS or CTS would have needed to traverse over multiple hops requiring complex handling. Similarly, the overhead of handling asymmetric links for routing protocols often justifies their avoidance altogether [19].

For simplicity we assume that network connectivity can be achieved without exceeding the maximum power capabilities of individual nodes. That is, each vertex representing a node in the induced graph potentially has edges to all other vertices. Our algorithms can easily be adjusted for a certain maximum power limit for the radios of each node.

\subsection{Classifications}

Based on the problem scenario $P \in\{P A G A, P A D A\}$, type of communication link $X \in\{D O, D D\}$ and cost metric $C \in\{\operatorname{Max} P$, Total $P\}$, our problems can be denoted as triplets of the form $\langle P, X, C\rangle$. For example $\langle P A G A, D O$, Max $P\rangle$ denotes the problem of power assignment with given antenna orientations for all nodes, having DO links, and seeking to minimize the MaxP cost metric. With two possible values for each field of the triplet, we have eight different problems in all.

3 Strong symmetry constraint requires that the communication graph contain only bidirectional links which is a stronger requirement than weak symmetry in which the communication graph can contain unidirectional links which, however, are not essential for connectivity. 


\section{Algorithms for PAGA Problem}

The power assignment algorithms we present in this paper all depend on constructing a minimum spanning tree (MST) on the complete graph formed between all nodes with edge weights equal to the minimum power required to communicate between them. So we start by describing MST construction for the two types communication links, DO and DD. Then we present our algorithms for the PAGA problem for both these types of links. We end the section by describing how the PAGA algorithms can be executed distributively.

\subsection{MST based Power Assignment}

The power required to communicate on the link between two nodes may be the same or different from either side of the link depending on the mode of communication. Thus, the MST construction varies depending on whether the nodes use DO or DD links. We will describe the simpler case of communicating with DD links first.

\subsubsection{MST for DD Links}

DD links use directional antennas at both ends of a link and the power required to communicate from one end point to the other is the same from either direction. Looking at Equation 1, this is because the gains in both directions are multiplied with each other. Thus the corresponding complete graph can be represented with undirected edges. Constructing an MST for an undirected graph can be done by using the prim's minimum spanning tree algorithm [20]. The power assignment for each node then is the maximum of all its adjacent edge weights in the resulting MST.

\subsubsection{MST for DO Links}

For DO links, only one end of a link uses a directional antenna. For example, consider two nodes of a link $u$ and $v$. The power required to communicate from $u$ to $v, \operatorname{Power}(u, v)$ depends on the distance between them and the gain of the directional antenna from $u$ to $v$ which in turn depends on the orientation of the antenna. Now even if the distance and power thresholds from both sides of the link are the same, the angle of antennas need not be. Thus the gain from $u$ to $v$ at antenna orientation $\Phi(u), g(u, v, \Phi(u)$ could be different than the gain in other direction from node $v, g(v, u, \Phi(v))$. Thus, the power required from both sides of the link could be different. An example of a complete directed graph on three nodes for DO links is shown in Figure 2a with powers required to 


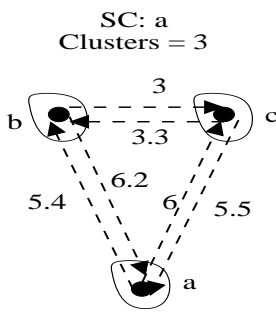

a)

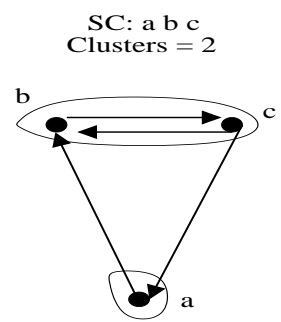

e)
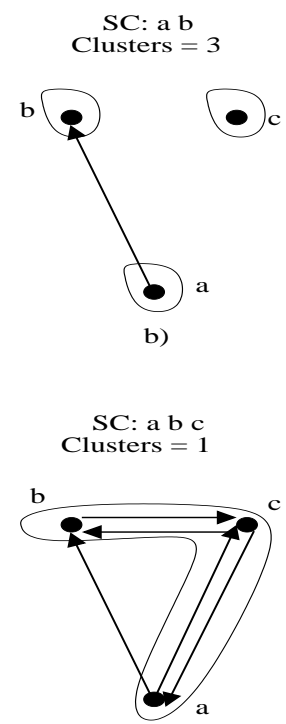

f)

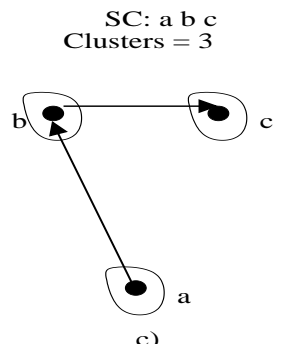

c)

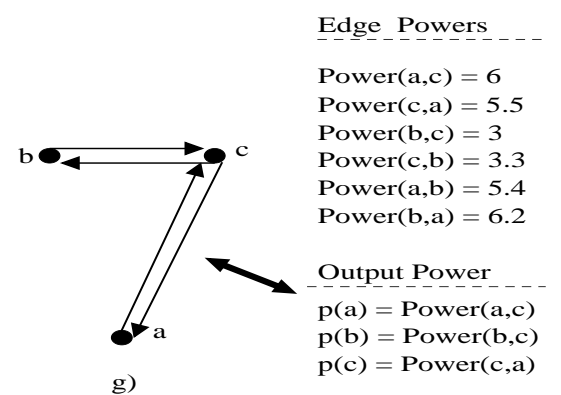

g)

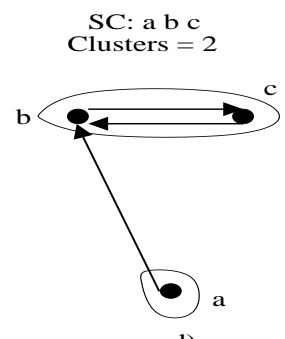

d)

Edge Powers

Power $(\mathrm{c}, \mathrm{a})=5.5$

Power(b,c) $=3$

Power $(b, a)=6.2$

p(a) $=$ Power $(\mathrm{a}, \mathrm{c})$

(c) $=$ Power $(\mathrm{c}, \mathrm{a})$

Fig. 2. Example showing step by step MST construction for a 3-node network with DO links

communicate between them. The MST construction proceeds (see Figure 2 for example) in a fashion similar to the prim's minimum spanning tree algorithm, however, with modifications to take care of directed edges between nodes. A semi-cluster $S C$ is initialized with the source node $a$. The role of $S C$ is to keep nodes which can be reached from the source using directed edges. When a new edge with least weight is sought, only those having at least one vertex in $S C$ are considered. When an edge is added from a node in $S C$ to one not in $S C$, the new node is added to $S C$. When adding an edge makes a link bidirectional or symmetric, the clusters of both end nodes are merged. This way, when only one cluster remains, the resulting graph can be pruned by removing asymmetric links to form the required MST with symmetric links. Note again that the power required on a link from one end to the other need not be the same in the other direction. The power assignment for each node then is the maximum of all its adjacent directed edge weights in the resulting MST.

\subsubsection{Post Processing}

After an MST based power assignment as described above, some nodes may be using up higher powers than required due to the presence of 'side-effect' edges. This phenomenon was first pointed out in [6]. An example for DD links is shown in Figure 3. An MST is constructed starting at source node $a$ and the final power assignment has the power of nodes $a$ and $d$ as, $p(a)=p(d)=$ Power $(a, d)$. However, when node $c$ adds an edge to $e$ and $b$ adds an edge to $f$, they use powers which are enough to communicate over link $(b, c)$ as well. Thus $a$ and $d$ can reduce their powers to a level just enough to reach $b$ 


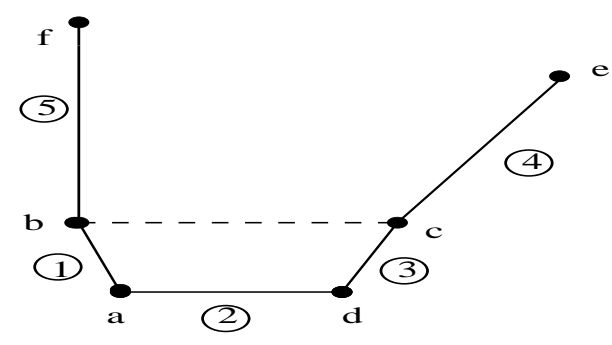

a)

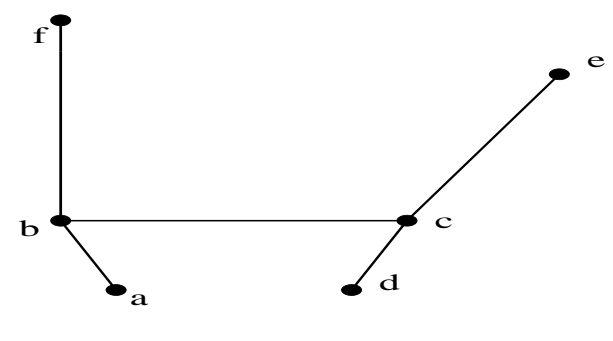

b)

Fig. 3. Phenomenon of side-effect edges in MST based power assignment. a) step by step construction of MST b)MST using smaller power at nodes $a$ and $b$

and $c$ removing edges $(a, d)$ and $(d, c)$. A simple binary search over all possible powers to other nodes can find the correct power level for each node that keeps the network connected. This post-processing has no effect on the MaxP metric but it does make a difference to the TotalP metric as it reduces the powers of some nodes. It also helps prune any asymmetric links using extra power at nodes, without affecting the connectivity property.

\subsection{Algorithm for DO Links}

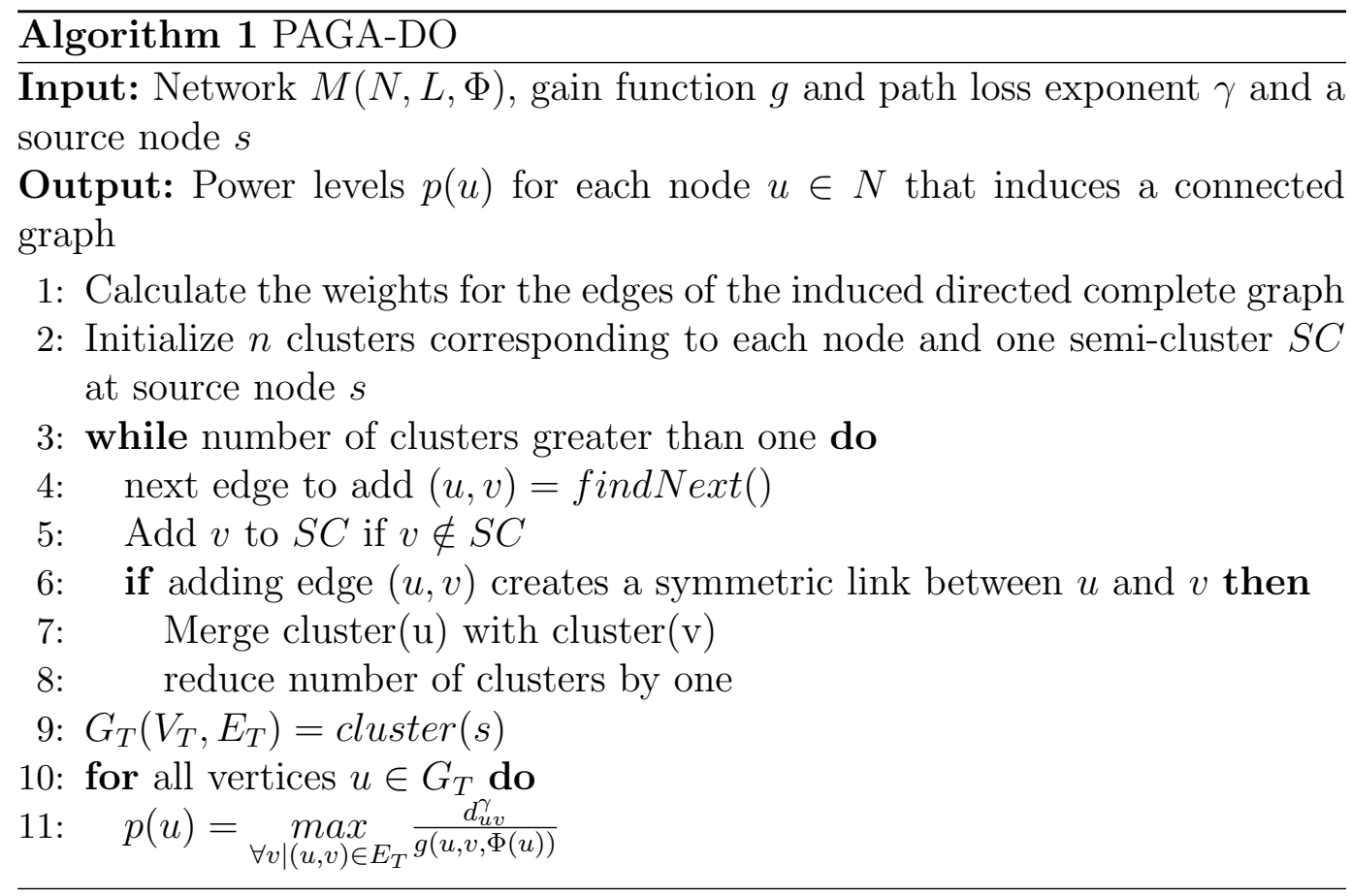

The PAGA-DO algorithm begins by calculating the gains of nodes towards each other using the gain function $g$ derived from Equations 1, 6 and 7. Information from $L$ and $\Phi$ is used to find the distances $d$ and angles of nodes towards each other for the required calculations to find the weights of the induced complete directed graph. The final power assignment for all nodes is 


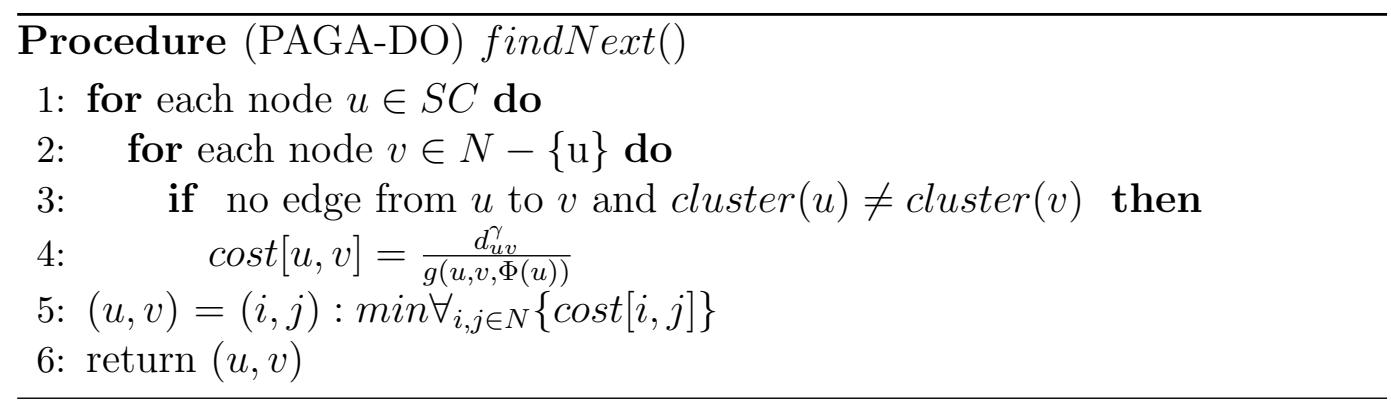

then found by constructing an MST as described in Section 4.1. The running time for the PAGA-DO algorithm is $O\left(n^{3}\right)$.

Theorem 4 PAGA-DO is an optimal algorithm for $\langle P A G A, D O, M a x P\rangle$.

PROOF. Line 4 adds a edge between two nodes if they are in different clusters. Lines 3 and 8 ensure that if we end, the graph is connected and all nodes have been considered.

Let the notation $p(u)$ denote the maximum power used by a node $u$. Assume the contrary that the maximum power of the network returned by our algorithm is not the optimum. Consider a node $u$ assigned the power $p(u)$. This has happened, by line 4 , when a new node $v$ was added to the cluster through $u$. Now since we are considering new nodes not in $u$ 's cluster which can be added with cheapest cost, there exists no path from $u$ to $v$ where all links along the path are symmetric.

$$
\nexists \operatorname{path}(u, v): \forall \operatorname{links}(x, y) \in \operatorname{path}(u, v), \exists \operatorname{edge}(x, y) \wedge \operatorname{edge}(y, x)
$$

This is because if all the links on any path from $u$ to $v$ were symmetric, we would have used that instead of trying to connect to $v$ directly from $u$.

Let the maximum powers of all nodes from the optimum algorithm be $p_{\text {opt }}(i)$. By our contrary supposition, the maximum power of optimal algorithm, $O P T=$ $\max _{\forall i}\left\{p_{\text {opt }}(i)\right\}<p(u)$. This gives us $p_{\text {opt }}(u)<p(u)$.

Now, if the above were to be true, it means that $u$ is not connected directly to $v$ and there was a path from $u$ to $v$. i.e.

$$
\exists \operatorname{path}(u, v): \forall \operatorname{links}(x, y) \in \operatorname{path}(u, v), \exists \operatorname{edge}(x, y) \wedge \operatorname{edge}(y, x)
$$

However, this is in contradiction to Equation 8. Hence, $p(u)=O P T$ and our algorithm returns the optimal maximum power.

Theorem 5 PAGA-DO is a 2-approximation algorithm for $\langle P A G A, D O$, TotalP $\rangle$

PROOF. It was proved by Althaus et al. that the power assignment derived 
from an MST is a 2-approximation to the problem ${ }^{4}$ of finding an optimal power assignment for the TotalP metric such that the network is connected with symmetric links [2]. The edge weights of the induced complete graph in this case were a function only of the distance between the corresponding two vertices (nodes). In the PAGA-DO algorithm, the edge weights are both a function of the distance as well as the gain of the transmitting node. Given a complete graph with these edge weights, the PAGA-DO algorithm also constructs an MST. Therefore the result follows for a power assignment derived from PAGA-DO.

\subsection{Algorithm for DD Links}

$\overline{\text { Algorithm } 2 \text { PAGA-DD }}$
Input: Network $M(N, L, \Phi)$, gain function $g$ and path loss exponent $\gamma$ and a
source node $s$

Output: Power levels $p(u)$ for each node $u \in N$ that induces a connected graph

1: Calculate the weights for the edges of the induced undirected complete graph

2: Initialize $n$ clusters corresponding to each node

3: Start from source node $s$

4: while Number of clusters greater than one do

5: $\quad$ next edge to add is $(u, v)=$ findNext ()

6: $\quad$ Merge cluster $(s)$ and cluster $(v)$

7: $\quad$ reduce number of clusters by one

8: $G_{T}\left(V_{T}, E_{T}\right)=\operatorname{cluster}(s)$

9: for all vertices $u \in G_{T}$ do

10: $\quad p(u)=\max _{\forall v \mid(u, v) \in E_{T}} \frac{d_{u v}^{\gamma}}{g(u, v, \Phi(u)) \cdot g(u, v, \Phi(v))}$

Procedure (PAGA-DD) findNext()

1: for each node $u \in \operatorname{cluster}(s)$ do

2: $\quad$ for each node $v \notin$ cluster $(s)$ do

3: $\quad \operatorname{cost}[u, v]=\frac{d_{u v}^{\gamma}}{g(u, v, \Phi(u)) \cdot g(u, v, \Phi(v))}$

4: $(u, v)=(i, j): \min \left(\forall_{i, j \in N} \operatorname{cost}[i, j]\right)$

5: return $(u, v)$

The power assignment by algorithm PAGA-DD is derived by constructing an MST from the induced undirected complete graph with the weights calculated in line 1. The algorithm is similar, but simpler than PAGA-DO due to the symmetry of DD links with the same running time of $O\left(n^{3}\right)$.

4 This problem was termed MIN-POWER SYMMETRIC CONNECTIVITY in the paper 
Theorem 6 PAGA-DD is an optimal algorithm for $\langle P A G A, D D, M a x P\rangle$.

PROOF. The proof is similar to that of Theorem 4.

Theorem 7 PAGA-DD is a 2-approximation algorithm for $\langle P A G A, D D$, TotalP $\rangle$.

PROOF. The proof is identical to that of Theorem 5.

\subsection{Distributed Execution of PAGA Algorithms}

As mentioned before, the PAGA algorithms are useful when a network exists with all nodes having fixed antenna orientations and the goal is to come up with a power-efficient topology. The algorithms will have more utility if they can be executed in a distributed fashion allowing nodes to find appropriate power levels for themselves. The main task would then be to construct an MST in distributed fashion after which nodes can assign themselves power levels equal to the maximum of their adjacent edge weights in the MST.

The distributed MST algorithm due to Gallager, Humblet and Spira can be utilized for this purpose [21]. We describe the algorithm briefly in the context of our wireless network setting and major changes required from the Prim's MST based algorithms presented above.

Each node is initially the root of its own cluster with all its adjacent edges unlabeled ${ }^{5}$. Then on, adjacent clusters join to form larger clusters by labeling their intermediate edge as a branch of the MST. The new branch is selected by the root of one or possibly both of the clusters as the minimum outgoing edge (MOE) for the entire cluster. This cluster MOE is determined by broadcasting an initiate message to all nodes of the cluster, requesting a report message with their local MOE to the root. Each node determines its local MOE by testing its unlabeled edges in order of non-decreasing weight until it finds one that leads to another cluster. Edges connecting nodes within the same cluster are labeled rejected and subsequently ignored. Each node gathers the reports of its children and reports the minimum MOE found by itself or its children. The root then sends a message changeRoot to the node adjacent to the MOE appointing it as the new leader of the cluster. The leader sends a connect message along the MOE and joins the cluster. The algorithm terminates when no node has any outgoing edges from it's cluster. The total message complexity of this distributed algorithm is $O\left(n^{2}\right)$.

For our setting, this distributed MST algorithm can be used as is for deriving

5 By adjacent edges, we mean the edges between a node and its neighbor at maximum power level. 
power levels for all nodes when using DD links. Modifications as described above (Section 4.1.2) for DO links can be done to take care of directed edges by ensuring that a cluster is connected through symmetric links. Moreover, a node at the other end of a MOE is declared a leader only if the MOE link is symmetric.

\section{Algorithms for PADA Problem}

In this section we present algorithms for the PADA problem to derive power levels as well as antenna orientations for all nodes. We begin by proving the PADA problem to be NP-complete and then present heuristic algorithms for solving this problem.

\subsection{Hardness results}

The PADA problem seeks to derive an antenna orientation as well as a power assignment for all nodes that minimizes the cost metrics while keeping the network connected. The task of finding both a power assignment and antenna orientation makes the problem difficult. We first prove the general decision version of PADA problem (without optimization as a goal) NP-Complete. This makes the problems that optimize for our cost metrics also NP-Complete.

This general decision version of the PADA problem can be stated as: Given a power assignment for all nodes, inducing a graph $G(V, E)$, is there an antenna orientation assignment such that the network is connected?

If we could solve the PADA decision problem in polynomial time, we could look at all the possible power assignments that matter ${ }^{6}$ and find the one that optimizes our cost metrics for the corresponding optimization problems and thus solve them in polynomial time.

Theorem 8 PADA is NP-Complete

PROOF. Given a power assignment and an antenna orientation for all nodes, we can check if the network is connected in polynomial time. Hence PADA $N P$.

We shall prove that PADA is NP-Hard by reducing from 3-SAT, a known NP-Complete problem. We will specifically look at the DO version only. The DD version can be proved similarly.

6 The number of power assignments that matter is $O\left(n^{2}\right)$ (hence, polynomial) since for each node we could possibly assign power levels to reach each of the other $n-1$ nodes 
Construct a graph $G$ with vertices equal to twice the number of clauses in the 3-SAT formula. Connect half of those vertices in a straight line with bi-directed edges between them forming a 'backbone' path. Then for each vertex of the backbone, connect a new terminal vertex corresponding to a clause. Each of these terminal vertices connect to their corresponding backbone vertex only if at least one literal of their corresponding clause is satisfied. For symmetry, a backbone vertex connects to its terminal vertex only if the same condition is satisfied. The construction is shown in Figure 4. Each variable's setting in the clause corresponds to a distinct antenna orientation as follows. For every variable $x_{i}, i=1$ to $n$, a setting of false corresponds to an orientation $2 i-1$ and a setting of true corresponds to an orientation of $2 i$.

Consider a simple 3-SAT example with two clauses: $\left(x_{1} \vee \bar{x}_{2} \vee x_{3}\right) \wedge\left(\bar{x}_{3} \vee x_{4} \vee \bar{x}_{5}\right)$. This corresponds to the case of two backbone vertices, $u$ and $v$, and two terminal vertices, $u^{\prime}$ and $v^{\prime}$ in the corresponding graph shown in Figure 5. Consider the first clause. To satisfy the first literal $x_{1}$, the antenna orientation has to be 2 (since $i=1$, and $2 i=2$ ). To satisfy the second literal $\bar{x}_{2}$, the antenna orientation has to be 3 (since $i=2$ and $2 i-1=3$ ). To satisfy the third literal $x_{3}$, the antenna orientation has to be 6 (since $i=3$ and $2 i=6)$. Thus, the terminal vertex $u^{\prime}$ is connected to the backbone vertex $u$ (and $u$ connects to $u^{\prime}$ by the symmetric construction described in previous paragraph) only if it uses an antenna orientation of 2,3 or 6. Similarly, the backbone vertex $v^{\prime}$ is connected to the terminal vertex $v$ (and $v$ to $v^{\prime}$ ) only if the antenna orientation at $v^{\prime}$ is 5,8 or 9 .

The claim is that with this construction, the graph $G$ will be connected for some antenna orientation setting for each vertex if and only if 3-SAT can be satisfied.

Now suppose, we have a satisfying assignment to the 3-SAT formula. Thus, every clause is satisfied and has at least one true literal. Correspondingly in the graph $G$, this means that all terminal vertices are connected to the backbone by at least one antenna orientation. This ensures that the graph is connected with symmetric links.

In the other direction, assume $G$ is connected with symmetric links. For this to happen, each terminal vertex must be connected to the backbone. Thus, there must be at least one orientation at which each terminal vertex is able to connect to the backbone. However, this orientation can be transformed to a boolean setting for a literal of the corresponding clause such that it is satisfied in the 3-SAT formula. Thus, each clause will evaluate to true, ensuring that the 3-SAT formula is satisfied.

We have proved $P A D A \in N P$ and also $P A D A$ is NP-Hard. Therefore, $P A D A$ is NP-complete.

Corollary $9\langle P A D A, D O, M a x P\rangle,\langle P A D A, D O$, Total $P\rangle,\langle P A D A, D D$, Max $P\rangle$ 


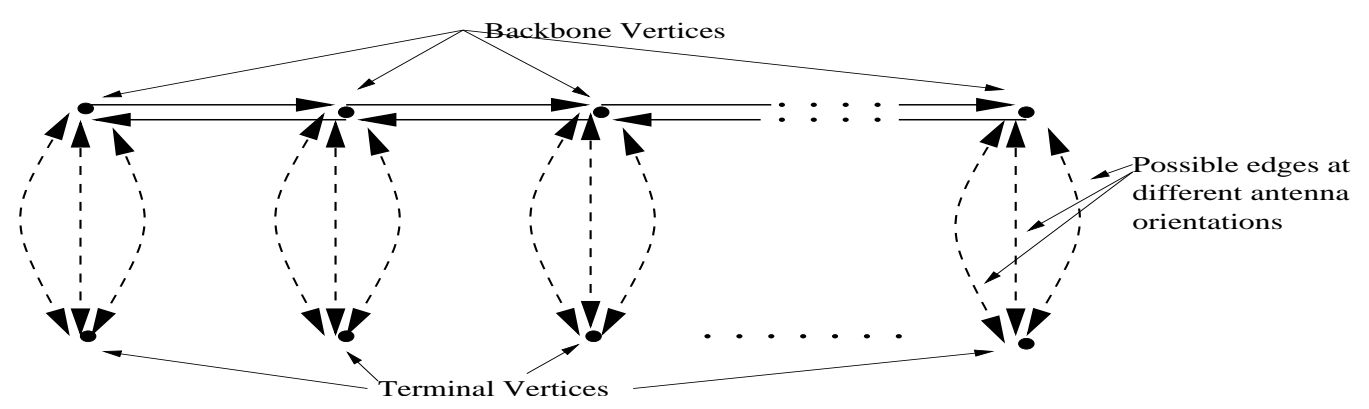

Fig. 4. Construction to prove PADA is NP-Complete

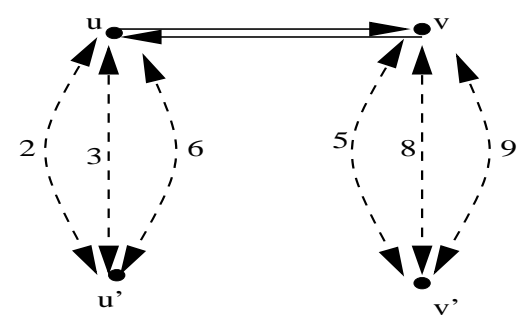

Fig. 5. Example construction with two clauses

and $\langle P A D A, D D$, TotalP $\rangle$ are NP-Hard.

PROOF. All these problems are optimization versions of the PADA decision problem. Since by Theorem 8 we have PADA to be NP-Hard, all these optimization problems are NP-Hard.

We now present heuristic algorithms with polynomial running times for the task of finding a power and orientation $(p, \Phi)$ assignment for all nodes.

\subsection{Algorithm for DO Links}

We can no longer pre-compute the weight of edges in constructing an MST as we did for the PAGA problems because of the variability of antenna orientation. Thus, the approach we take is to construct an MST, edge by edge, adding directed edges till we have a connected graph of symmetric links. At each stage of checking which edge can be added most cheaply, a node finds the best angle at which this new edge could be added using up its least power. Since a node's assigned power is the maximum over all its adjacent edges, a min max optimization problem needs to solved to find the correct orientation.

A new edge is selected to be added to SC based on the procedure findNext. The algorithm builds up its cluster from the source node $s$ and adds a new edge to the SC based on which edge can be added most cheaply. The 'best' 


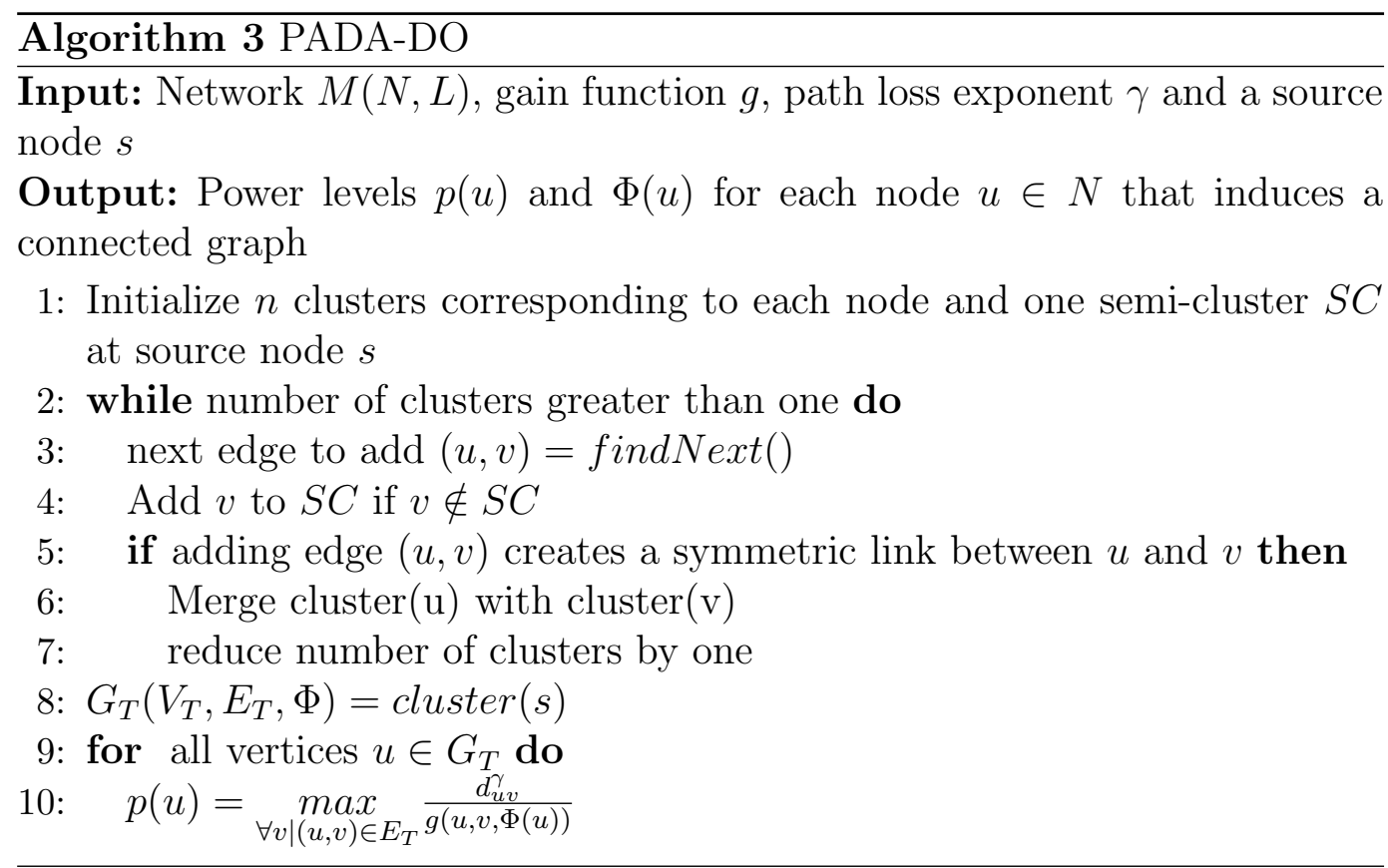

\section{Procedure(PADA-DO) findNext ()}

1: for each node $u \in S C$ do

2: $\quad$ for each node $v \in N-\{u\}$ do

3: $\quad$ if no edge from $u$ to $v$ and $\operatorname{cluster}(u) \neq \operatorname{cluster}(v)$ then

4: $\quad\langle\operatorname{mincost}[u, v], \phi[u, v]\rangle=\operatorname{findMinCost}(u, v, N \operatorname{NrList}(u))$

5: $(u, v)=(i, j): \min \forall_{i, j \in N}\{\operatorname{mincost}[i, j]\}$

6: $\Phi(u)=\phi[u, v]$

7: return $(u, v)$

Procedure(PADA-DO) findMinCost $(u, v, N$ brList $(u))$

1: $N(u)=\operatorname{NbrList}(u) \cup\{v\}$

2: $\min _{\forall \in \in N(u)} \max \left\{\frac{d_{u w}^{\gamma}}{g(u, w, \phi)}\right\}$, with $\phi \in[0, \theta]$

3: $\operatorname{cost}=\max _{\forall w \in N(u)} \frac{d_{u w}^{\gamma}}{g(u, w, \phi)}$

4: Store optimal angle orientation $\phi[u, v]=\phi$ for adding $v$ through $u$

5: return cost

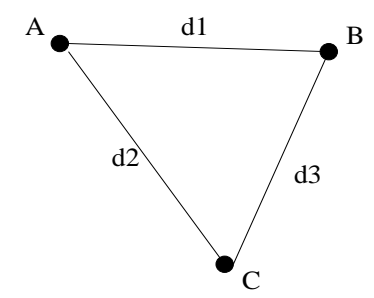

$\mathrm{d} 1<\mathrm{d} 2<\mathrm{d} 3$

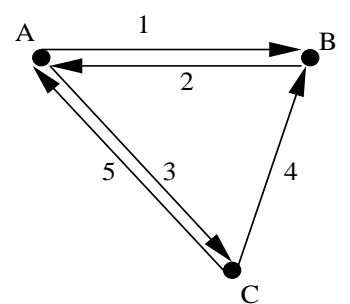

Fig. 6. PADA example showing step by step construction for a 3-node network with DO links 
edge added to SC through a node $u \in S C$ is one that co-exists best (i.e minimizes maximum power used by any adjacent edge of $u$ ) with the other already selected adjacent edges of $u, N b r \operatorname{List}(u)$. Thus, when a new edge $(u, v)$ is tried out by a node $u \in S C$, the maximum cost it incurs for connecting to all nodes in $N b r \operatorname{List}(u)$ and the new node $v$, is calculated with the best possible antenna orientation of $u$, say $\phi$. The procedure findMinCost () does this by first finding the best $\phi$ by non-linear programming (NLP). From Equation 6 we have, $g(u, w, \phi)=G_{m a x} \cdot e^{\frac{-\left(\theta_{u w}-\left(\phi-\theta_{m}\right)-\theta_{m}\right)^{2}}{2 \sigma^{2}}}=G_{m a x} \cdot e^{\frac{-\left(\theta_{u w}-\phi\right)^{2}}{2 \sigma^{2}}}$ where $\theta_{u w}-$ $\left(\phi-\theta_{m}\right)$ is the angle from node $u$ to $w$ within the beamwidth ${ }^{7}$.

Thus the NLP problem can be formulated as

$$
\min _{\forall} \max \left\{\frac{d_{u, w(u)}^{\gamma}}{G_{\text {max }} \cdot e^{\frac{-\left(\theta_{u w-\phi)^{2}}\right.}{2 \sigma^{2}}}}\right\}, \phi \in[0, \theta]
$$

$\phi$ is the only variable in Equation 10. All the other parameters can be precomputed based on location and antenna beamwidth information.

This orientation $\phi$ is then used to find the power used by $u$ after adding $v$. Once the algorithm finally decides that the next edge to be added is from $u$ to $v$, it commits $\Phi(u)=\phi[u, v]$ (line 6 of findNext()). No antenna orientation information is used for gain calculations at the beginning of the algorithm, because the antenna orientation of nodes is varied as the algorithm proceeds to arrive at the final heuristic solution.

Figure 6 shows an example of the way the PADA-DO algorithm proceeds. Nodes A, B and C are separated by distances as shown. Node A, the source node, starts by adding $\mathrm{B}$ to the semi-cluster $\mathrm{SC}$ as $\mathrm{B}$ is the node that can be added with least power. This is because $d_{1}$ is shortest and also because $\mathrm{A}$ can orient itself towards B providing maximum gain in that direction. The next cheapest edge to be added is BA with a similar argument as above. Now both $\mathrm{A}$ and $\mathrm{B}$ form a cluster with a symmetric link between them. The task now is to form a symmetric link from $\mathrm{C}$ to any of these two to form the required connected graph with symmetric links. The algorithm then finds what is best orientation at which $\mathrm{A}$ can add $\mathrm{C}$ (along with existing edge to $\mathrm{B}$ ) and keep its power minimum. $\mathrm{B}$ does similarly. The node that will require minimum power among these adds the edge to $\mathrm{C}$. In this case $\mathrm{A}$ requires the minimum power to add $\mathrm{C}$. Node $\mathrm{C}$ then adds an edge to $\mathrm{B}$ as $d_{2}$ is lesser than $d_{3}$ and its antenna has no other edges to care about. But since the network is still not connected by symmetric links, $\mathrm{C}$ has to add an edge to A before the algorithm terminates.

Now we look at how accurate we can expect the heuristics presented above to be. For that, we look at the scenario where our algorithm can go wrong compared to an optimal algorithm. Figure 7 shows an example with a 5-node

7 This corresponds to $\theta_{k}$ in Figure 1 where we now subtract out the angle between positive $\mathrm{x}$-axis and beginning of the beam to find out angle from $u$ to $w$ within the beam 


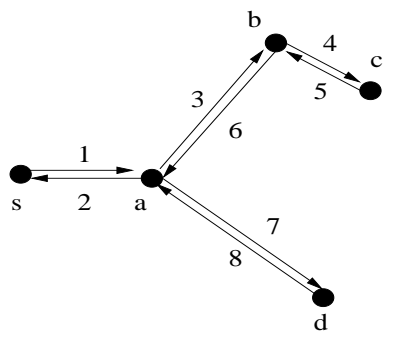

a)

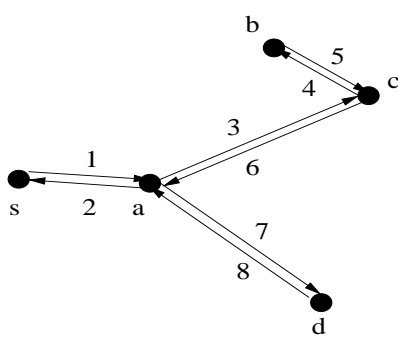

b)

Fig. 7. Example showing worst case for PADA-DO through a step by step construction for a 5-node network. Distances between node pairs: $d_{s a}=2, d_{a b}=3.1, d_{a c}=3.2, d_{a d}=3.3, d_{b c}=1$, all other $d_{x x}>5$. Step numbers are shown alongside each directed edge. a) Topology for PADA-DO algorithm b) Topology for optimal algorithm

network. We start with a source node $s$ and add a directed edge to $a$ because it is the cheapest cost edge. Similarly $a$ adds a directed edge to $s$. The labels on edges indicate the step at which they were added. In step 3, a has to decide whether to add a directed edge to $b, c$, or $d$. It chooses the former as it finds that retaining edges $(a, s)$ and $(a, b)$ keeps its power minimum compared to any other combination. Next $b$ and $c$ add edges to each other, followed by an edge back to $a$ from $b$. Now it remains to add $d$ to the network to make it connected. In the next two steps edges are added between $a$ and $d$ as this was found to be the most economical way to add $d$ after all other edges were added. Our algorithm could go wrong in the above series of steps at the point where $a$ has to decide between $b, c$ and $d$. $b$ was added because it was cheapest according to $a$ at that point. However, later on when $d$ was added, it could have been the case that edges $(a, s),(a, c)$ and $(a, d)$ together resulted in a smaller power at $a$ than edges $(a, s),(a, b)$ and $(a, d)$. Of course, there was no way of $a$ knowing beforehand that such an edge $(a, d)$ would be added later, something which an optimal algorithm would have found. In the worst case, the optimal algorithm would have assigned the peak gain of $G_{\max }$ towards edge from $a$ to $d$ while our algorithm might have assigned some gain $G_{l b}, G_{\min } \leq G_{l b} \leq G_{\max }$.

Theorem 10 PADA-DO is a $\frac{G_{\max }}{G_{\min }}$-approximation algorithm for $\langle P A D A, D O, \operatorname{Max} P\rangle$.

PROOF. Ratio of maximum power used by PADA-DO algorithm to that of optimum algorithm for the edge $u, v$ which requires the maximum power is $\frac{d_{u v}^{\gamma}}{G_{l b}} / \frac{d_{u v}^{\gamma}}{G_{\max }}=\frac{G_{\max }}{G_{l b}} \leq \frac{G_{\max }}{G_{\min }}$

Theorem 11 PADA-DO is a 2. $\frac{G_{\max }}{G_{\min }}$-approximation algorithm for $\langle P A D A, D O$, TotalP $\rangle$.

PROOF. As shown above, each node's power can exceed the optimal of an 
MST based power assignment by at most a factor of $\frac{G_{\max }}{G_{\min }}$. Hence, the total power of all nodes can exceed the optimal by at most a factor of $\frac{G_{\max }}{G_{\min }}$. From Theorem 5, since an MST based construction is a 2-approximation for TotalP metric, PADA-DO is a $2 \cdot \frac{G_{\max }}{G_{\min }}$-approximation to $\langle P A D A, D O$, TotalP $\rangle$.

\subsection{Algorithm for DD Links}

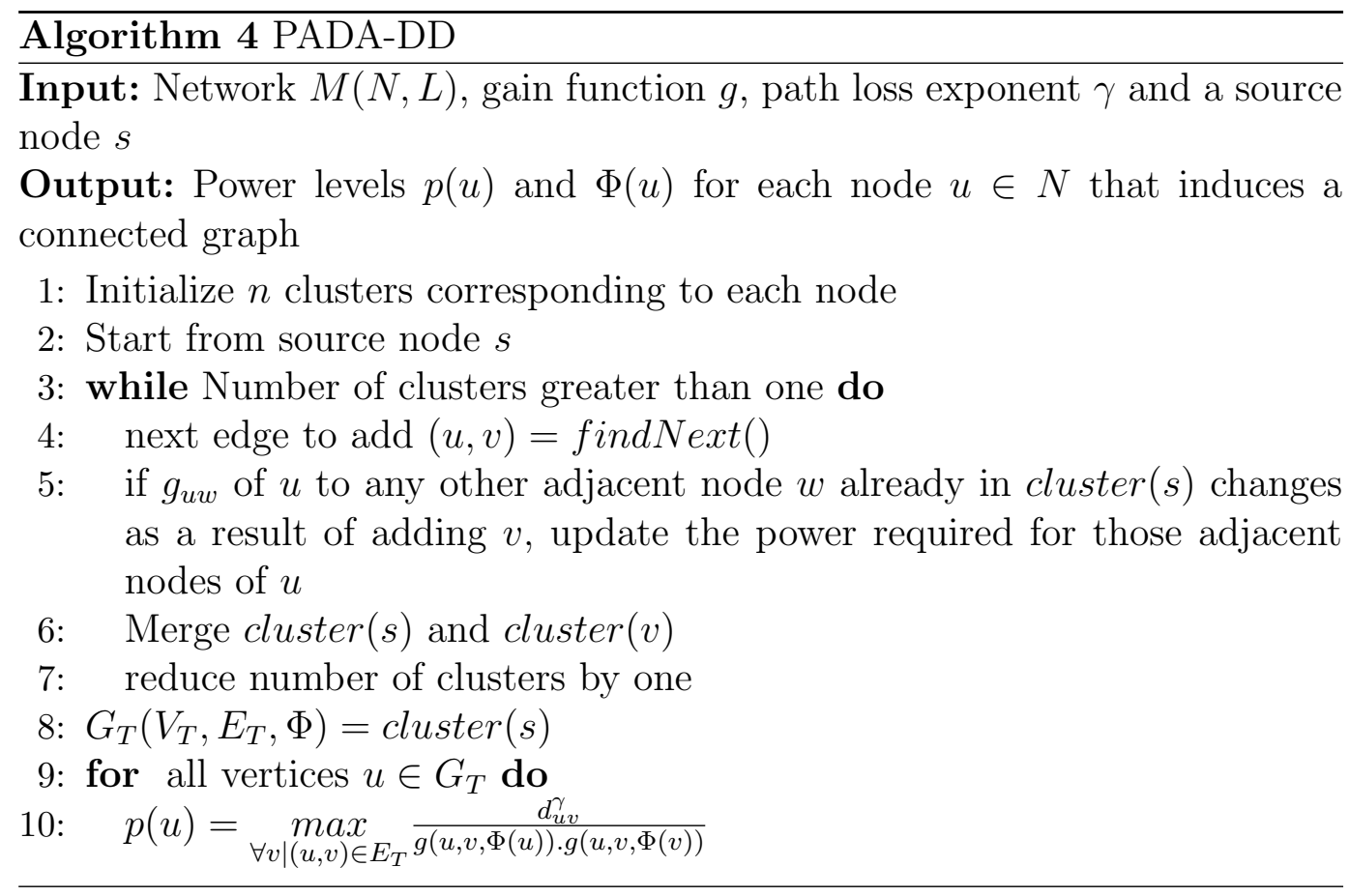

Procedure(PADA-DD) findNext()

1: for each node $u \in$ cluster $(s)$ do

2: $\quad$ for each node $v \notin$ cluster $(s)$ do

3: $\quad\langle\operatorname{mincost}[u, v], \phi[u, v]\rangle=\operatorname{findMinCost}(u, v, \operatorname{Nbr} \operatorname{List}(u))$

4: $(u, v)=(i, j): \min \left(\forall_{i, j \in N} \operatorname{mincost}[i, j]\right)$

5: $\Phi(u)=\phi[u, v]$

6: return $(u, v)$

Procedure(PADA-DD) findMinCost(u,v,NbrList(u))

1: $N(u)=\operatorname{NbrList}(u) \cup\{v\}$

2: $\min _{\forall} \max \left\{\frac{d_{u, w}^{\gamma}}{g(u, w, \phi) \cdot g(w, u, \Phi(w))}\right\}$, s.t $\phi \in[0, \theta]$

3: $\operatorname{cost}=\max _{\forall w \in N(u) \cup\{v\}} \frac{d_{u, w}^{\gamma}}{g(u, w, \phi) \cdot g(w, u, \Phi(w))}$

4: Store optimal angle orientation $\phi[u, v]=\phi$ for adding $v$ through $u$

5: return cost

The PADA-DD algorithm has a major difference with the PADA-DO version. The gains are calculated between nodes as before, but the power required now 
depends on gains of both end nodes of a link. Thus the power required is symmetrical and the algorithm does not have to deal with directional edges as explained before in Section 4.1.

The next node to be added is found in a similar fashion to PADA-DO. Since $e^{-\left(\theta_{u w}-\phi\right)^{2}} \cdot e^{-\left(\theta_{w u}-\Phi(w)\right)^{2}}=e^{-\left(\theta_{u w}-\phi\right)^{2}-\left(\theta_{w u}-\Phi(w)\right)^{2}}$, the NLP problem can be formulated as

$$
\min _{\forall \in \in N(u)} \max \left\{\frac{d_{u, w}^{\gamma}}{G_{\text {max }}^{2} \cdot e^{\frac{-\left(\theta_{u w}-\phi\right)^{2}-\left(\theta_{w u}-\Phi(w)\right)^{2}}{2 \sigma^{2}}}}\right\}, \phi \in[0, \theta]
$$

The $\Phi(w)$ of a new vertex $w$ to be added through a vertex $u$ is taken as the optimal orientation which assigns maximum gain in the direction of $u$. A new vertex added to a cluster does not have any orientation of its own and thus can be assigned any orientation.

One important thing to be taken care of is that when a new node $v$ is added to cluster $(s)$, it is likely that the node $u$ in cluster $(s)$, through which $v$ was added, will change its orientation and thus gain. Since the links are DD, all adjacent nodes of $u$ will have to re-adjust their powers to reach $u$ and viceversa (line 5 of Algorithm 4).

Next we prove the accuracy of the heuristics for DD case in a similar fashion to that of the DO case.

Theorem 12 PADA-DD is a $\left(\frac{G_{\max }}{G_{\min }}\right)^{2}$-approximation algorithm for $\langle P A D A, D D, \operatorname{Max} P\rangle$.

PROOF. Ratio of maximum power used by PADA-DD algorithm to that of optimum algorithm for the edge $u, v$ which requires the maximum power is $\frac{d_{u v}^{\gamma}}{G_{l b} \cdot G_{l b}} / \frac{d_{u v}^{\gamma}}{G_{\max } \cdot G_{\max }}=\left(\frac{G_{\max }}{G_{l b}}\right)^{2} \leq\left(\frac{G_{\max }}{G_{\min }}\right)^{2}$.

Theorem 13 PADA-DD is a 2.( $\left.\frac{G_{\max }}{G_{\min }}\right)^{2}$-approximation algorithm for $\langle P A D A, D D$, Total $P\rangle$.

PROOF. As shown above, each node's power can exceed the optimal of an MST based power assignment by at most a factor of $\left(\frac{G_{\max }}{G_{\min }}\right)^{2}$. Hence, the total power of all nodes can exceed the optimal by at most a factor of $\left(\frac{G_{\max }}{G_{\min }}\right)^{2}$. From Theorem 7, since an MST based construction is a 2-approximation, PADA-DD is a 2. $\left(\frac{G_{\max }}{G_{\min }}\right)^{2}$-approximation to $\langle P A D A, D D$, Total $P\rangle$. 

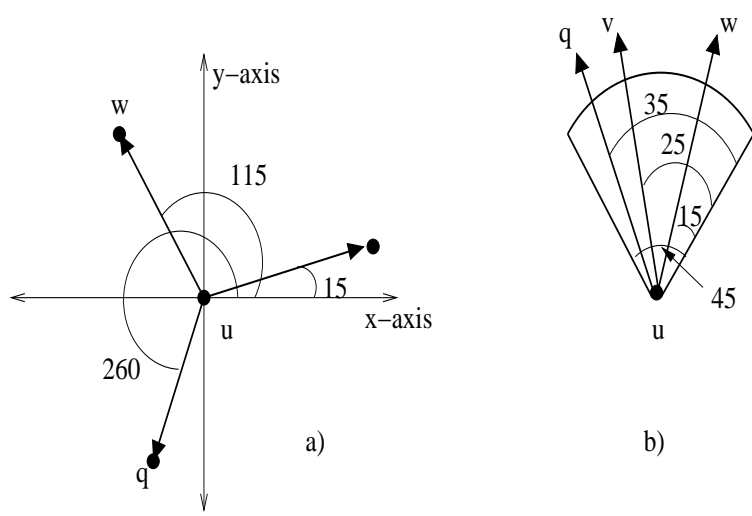

Fig. 8. Example mapping of various angles to a single beam a) angles of neighbors b) translation to a single beam

\section{Analysis of worst-case gain by heuristic algorithms}

We used the fact above that a lower bound on the gain assigned by our heuristic algorithms obeys $G_{\min } \leq G_{l b} \leq G_{\max }$ and gave approximation ratios based on the lower bound. However, it can be shown that $G_{l b}>G_{\text {min }}$ which improves our approximation ratios. We develop the theory below. The angles of a node towards its neighbors varies from 0 to $360^{\circ}$. These edges may lie on different beams of the node. The position or angle of these edges on a beam decides the gain in that direction. It can be easily visualized by mapping all edges to a single beam. Let $\alpha_{u v}$ denote the angle of an edge $(u, v)$ from the positive $\mathrm{x}$-axis of $u$ in anti-clockwise direction. Then, given all such $\alpha_{u v}$ corresponding to all neighbors $v$ of $u$, we can map them within a beam as $\theta_{u v}=\alpha_{u v} \bmod \theta$, where $\theta_{u v}$ is the angle of edge $(u, v)$ within a beam of beamwidth $\theta$. A sample mapping is shown in Figure 8.

The min max optimization NLP we solved before gave us an antenna orientation with maximum gain possible to all edges of a node such that the maximum of powers used by its edges is minimum. The worst-case scenario occurs when all neighbors of a node are equi-distant. If not, the NLP solution would find an orientation which has higher gain towards the edge using maximum power, as this will minimize the power of the node. We want to find what is the worst possible gain, $G_{l b}$, an edge responsible for maximum power at a node could be assigned under the circumstances. This worst case gain would give us a bound on maximum power of a node in terms of its longest edge.

Define radial distance as the distance between the points of intersection of edges (mapped to a single beam) and the curved part of a beam (see Figure 9a). Given any mapping of edges to a beam such that the neighboring nodes corresponding to these edges are equi-distant, the NLP tries to find an antenna orientation that pushes all the edges to the center of the beam as much as possible. Figure 9 shows some examples. Let $k$ be the largest radial distance between two successive points of intersection, which include the two endpoints of the beam (see Figure 9b, c, d). Let $l$ be the radial distances of the first point 


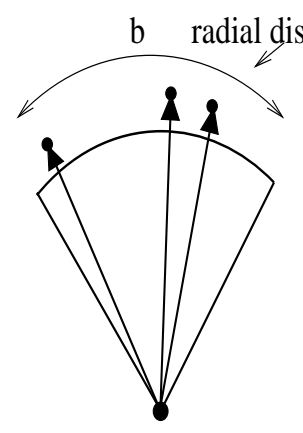

a)

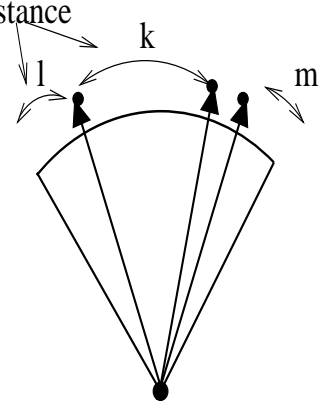

b)

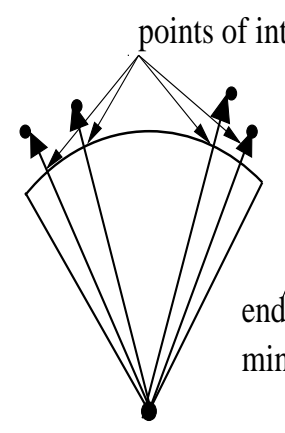

c)

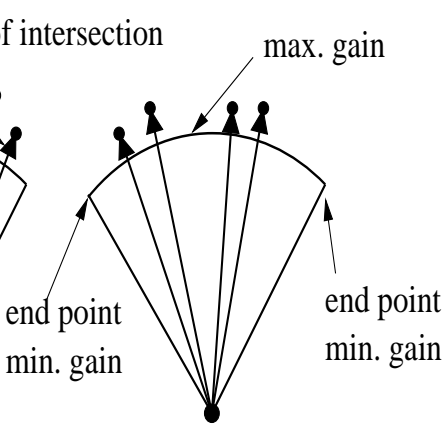

d)

Fig. 9. Two examples of adjustment of antenna orientation to minimize maximum power. Some key terms are also labeled. $(\mathrm{a}->\mathrm{b})$ antenna orientation is adjusted to average of radial distances of end points $(l+m) / 2$. (c->d) antenna orientation is adjusted to center of largest radial separation $k$

on the left from the left end point and $r$ the corresponding point on the right side.

Lemma 1 The radial distance of an edge from the points of minimum gain with the antenna orientation derived from solving $N L P$ is at least $\max \left(\frac{k}{2}, \frac{l+m}{2}\right)$.

PROOF. This can be shown with a geometrical argument. We can position the antenna such that the two intersection points corresponding to $k$ lie on either side of the point of minimum gain such that each edge is at a distance $k / 2$. Or we could position the antenna such that we average out the distances $l$ and $m$ of edges from the end points to maximize the distance of the one nearest the end point to make $l=m=\frac{l+m}{2}$. We can choose the maximum of these two choices to maximize the gain of the edge with least gain. The transitions from $a$ to $b$ and from $c$ to $d$ of Figure 9 show these two possibilities.

It remains to see what the smallest possible value of $\max \left(\frac{k}{2}, \frac{l+m}{2}\right)$ is. This case occurs when we have $t$ intersection points uniformly spaced on the radial perimeter $b$ of a beam. With $l=m=k / 2$, we have $2 \frac{k}{2}+(t-1) k=b$, solving which we get $k=\frac{b}{t}$. Using Lemma 1 the minimum radial distance from any side is thus $k / 2=\frac{b}{2 t}$. Geometrically, this radial distance can be transformed to an angle $\theta_{l b}=\frac{\theta \cdot(b / 2 t)}{b}=\frac{\theta}{2 t}$. Thus, the minimum gain is

$$
G_{l b}=g\left(\theta_{l b}\right)=g\left(\frac{\theta}{2 t}\right)
$$

The greater $t$ is, smaller is our guaranteed minimum gain, requiring larger powers. The following lemma bounds the maximum possible value of $t$.

Lemma 2 The number of neighbors of any node is no more than $6 . \frac{G_{\max }}{G_{\min }}$. 


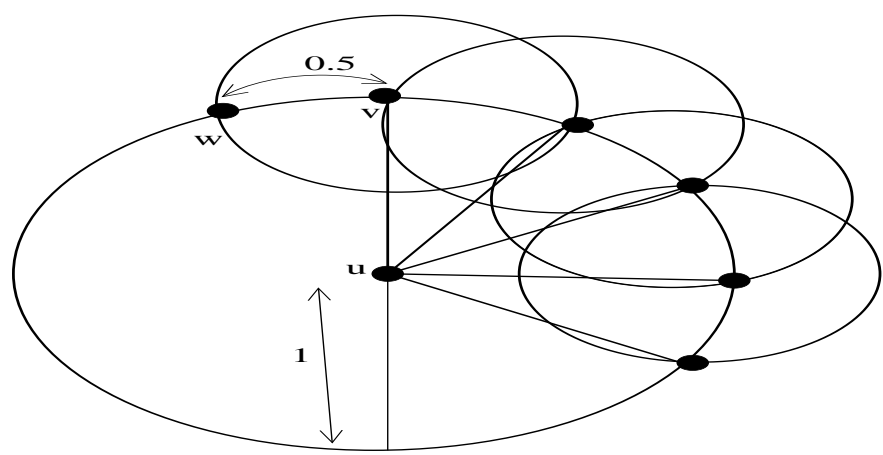

Fig. 10. Bounding number of neighbors of a node in an MST on a plane. In example, radius of larger circle is unity and that of smaller circle is $G_{\min } / G_{\max }=0.5$

PROOF. It is well known that in an MST $T$ on the euclidean plane, any vertex has degree no more than 6 [22]. We extend this result for the case where each edge is influenced by gain of antennas and we seek the maximum number of neighbors possible for a node with all its neighbors equi-distant.

Given distances $d_{u v}$ and $d_{u w}$ (and corresponding powers $p($.$) ) between a node$ $u$ and two other vertices $v$ and $w$, edges $(u, v)$ and $(u, w)$ exist in $T$ only if $p_{v w}>p_{u v}$ and $p_{v w}>p_{u w}$. In the worst case, edge $(v, w)$ may have a gain of $G_{\max }$, while edges $(u, v)$ and $(u, w)$ may only have gains of $G_{\min }$. Thus, $w$ cannot be within $\frac{d_{v w}^{\gamma}}{G_{\max }}$ of $v$. That is, with $p_{u v}=p_{u w}$ scaled to $1, p_{v w}$ has to be greater than $\frac{G_{\min }}{G_{\max }}$. Again using elementary geometry, we have total length of circumference $=2 \pi .1=2 \pi$. Each neighbor of $u$ has to be at least $\frac{G_{\min }}{G_{\max }}$ apart from any other neighbor. Thus, total number of possible neighbors is

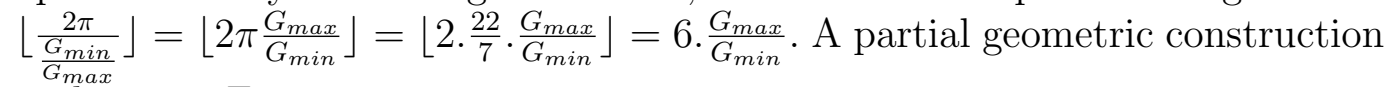
is shown in Figure 10.

Theorem 14 The minimum gain towards an edge of a node that costs maximum power is at least $G_{l b}=g\left(\frac{\theta}{12} \cdot \frac{G_{\min }}{G_{\max }}\right)$.

PROOF. This result follows directly from Equation 12 and Lemma 2.

We can thus replace $G_{\min }$ by $G_{l b}$ in Theorems 10 to 13 with $G_{l b}>G_{\text {min }}$ leading to better approximation bounds. In the evaluation section we demonstrate that the power consumption of our heuristic algorithms are much better than the stated approximation bounds.

\section{Evaluation}

We aim to demonstrate through our simulation based evaluation, the benefits of making antenna orientation a parameter in topology construction, for both 
the MaxP and TotalP metrics, by comparing the PAGA and PADA algorithms for both DO and DD links. As described before, the PAGA scenario uses a given fixed antenna orientation and can be used to represent algorithms which do not use antenna orientation as a parameter. Further, the effect of antenna beamwidth on power savings by our algorithms is quantified. We end the section by comparing the power efficiency of our heuristic algorithms with optimal values.

\subsection{Experimental Setup}

In the simulation experiments, the nodes were uniformly distributed within an area of $100 \times 100$. Each data point shown is the average of 100 runs. Unless specified otherwise, a beamwidth of $30^{\circ}$ is used. For PAGA experiments, each node is given an antenna orientation of $0^{\circ}$ (refer Figure 1 ). The randomness in the locations of nodes is enough to provide randomness in gains of node pairs towards each other. $95 \%$ confidence intervals for the mean are shown where

applicable. The expression $G_{\max }=\frac{2 \pi}{\theta}$, modeling the gain of antenna along the azimuthal plane, was used to calculate the value of $G_{\max }$ for various values of beamwidth.

Beginning with a complete graph on 50 nodes, the derived topologies from the four algorithms are shown in Figure 11 for a sample run of the simulation. This gives an idea of the 'thinning' of the topology relative to the original graph. The darker lines for algorithms with DO mode are due to bi-directed edges. For comparisons between the algorithms in terms of power used, we need to rely on the subsequent experiments and their results.

\subsection{Power Consumption}

Here we look at the power consumption of the algorithms PAGA-DO, PAGADD, PADA-DO and PADA-DD for metrics MaxP and TotalP at various node densities. The difference between the power consumption of PAGA and PADA algorithms demonstrates the benefit of building networks making antenna orientation a parameter. Note that since the PADA algorithms are only heuristics, the benefits shown are only a lower bound.

Figure 12 shows the maximum transmit power (MTP) for all four algorithms. As expected, the MTP required decreases with increasing density. The higher power required for DO links compared to DD links follows Equations 2-5. More important, however, is the reduction in MTP achieved by using the antenna orientation as a parameter in case of PADA algorithms. Since the reduction is not easy to make out in Figure 12, the corresponding reductions as a percentage are shown in Figure 14. The power reductions are quite consistent across 


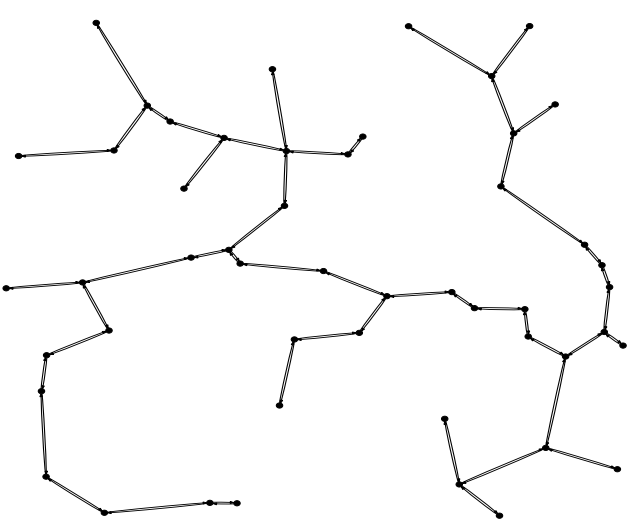

(a) Topology due to PAGA-DO

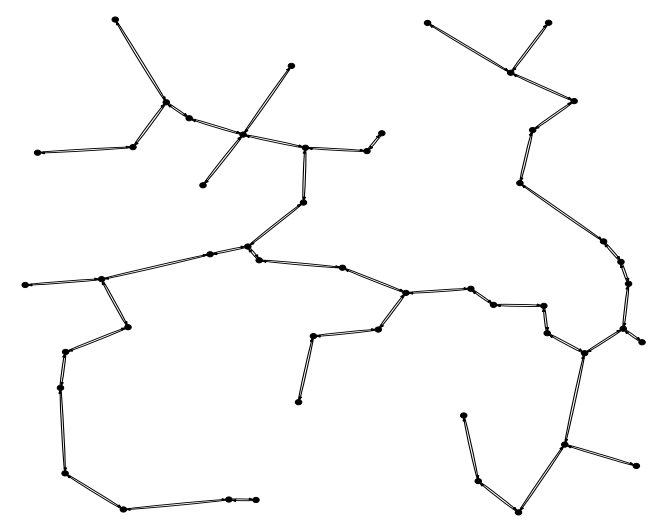

(c) Topology due to PADA-DO

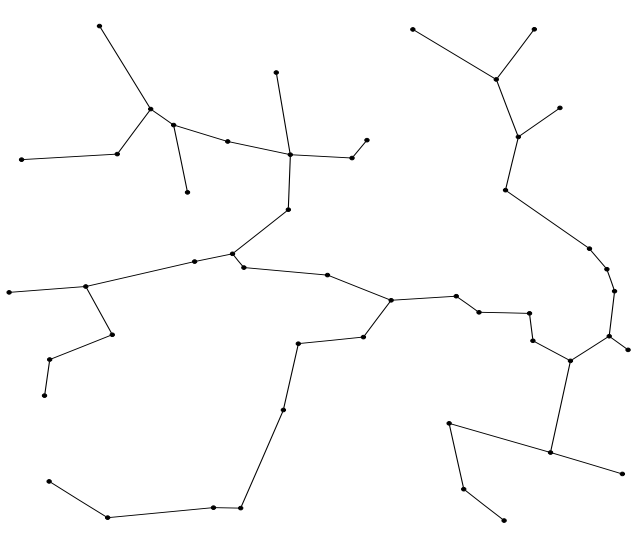

(b) Topology due to PAGA-DD

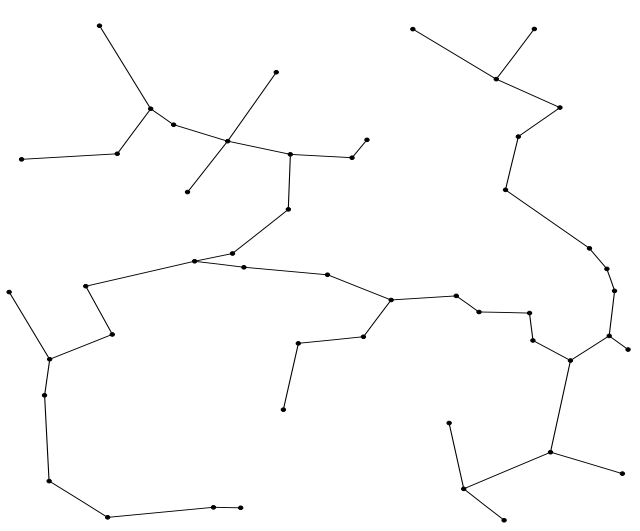

(d) Topology due to PADA-DD

Fig. 11. Controlled topologies with the four algorithms

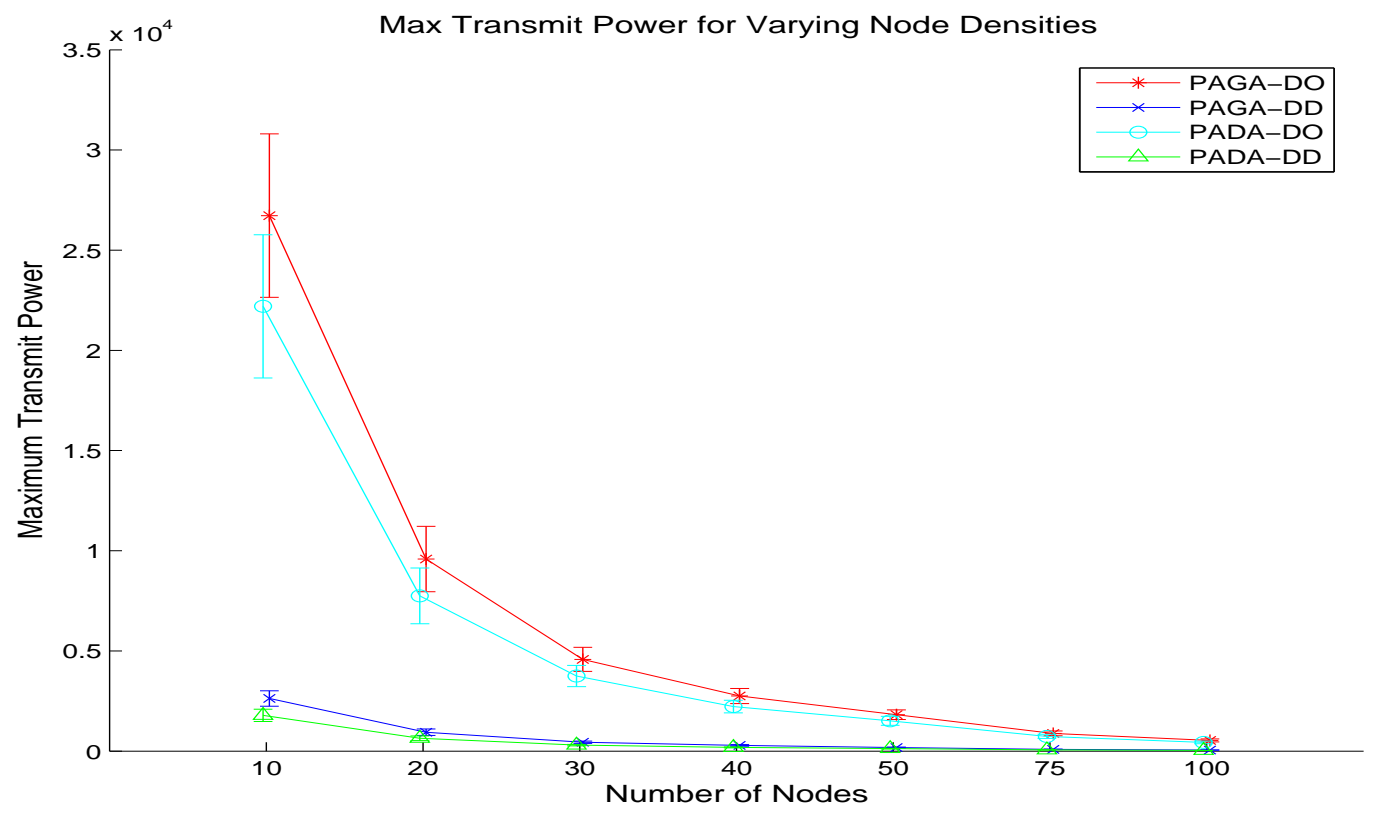

Fig. 12. Comparing maximum transmit power required by all schemes over different node densities 


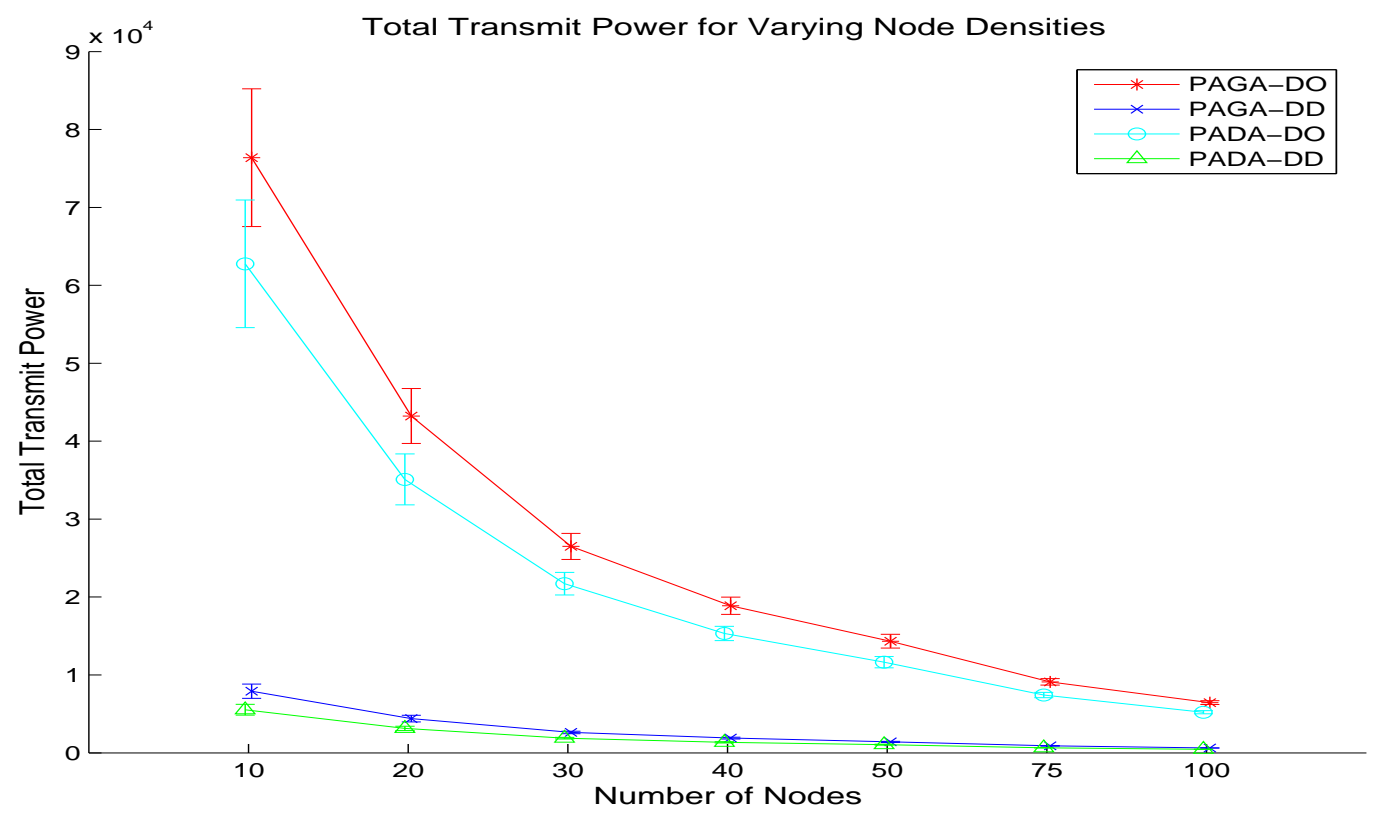

Fig. 13. Comparing total transmit power required by all schemes over different node densities

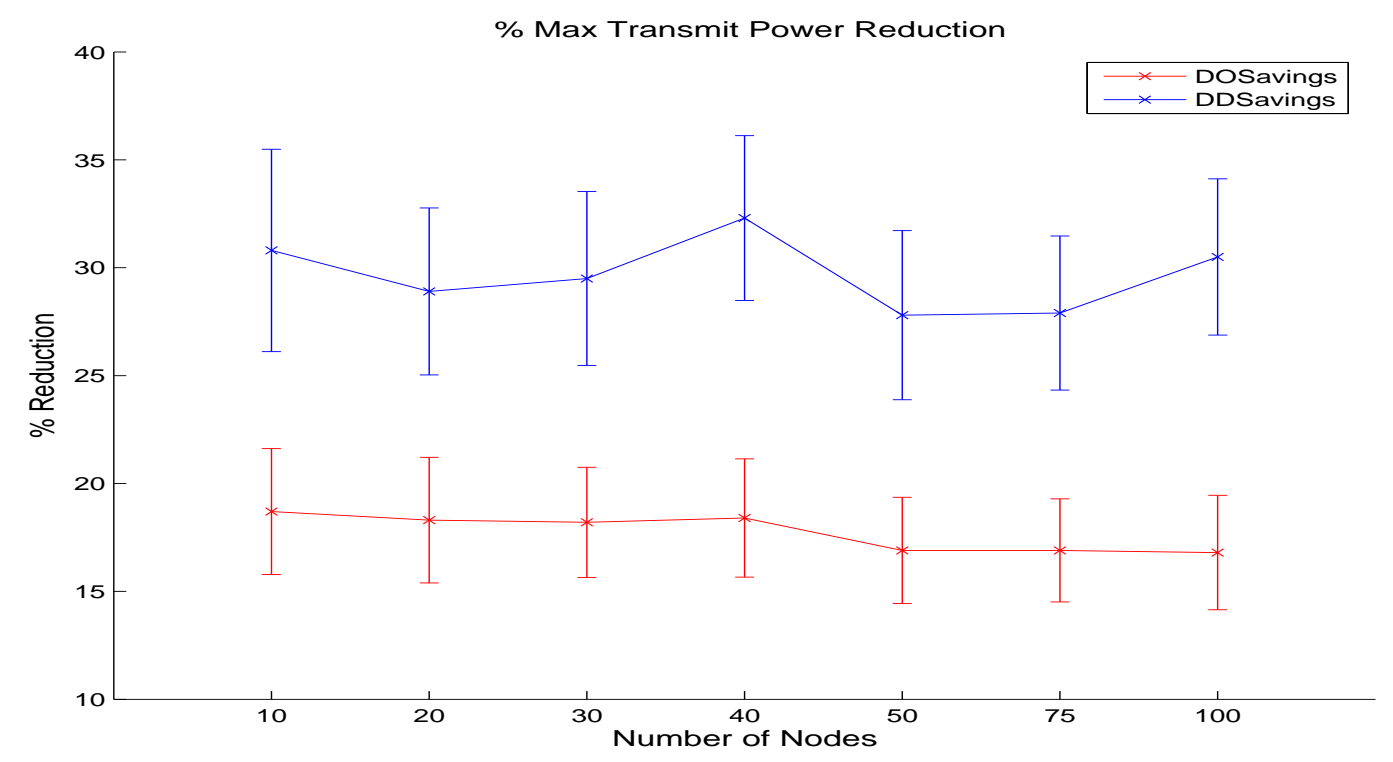

Fig. 14. Percentage savings by using antenna orientation as a parameter for MaxP metric

various densities with averages of about $18 \%$ and $30 \%$ for the DO and DD case respectively. A very similar trend is observed in results for the TotalP metric shown in Figures 13 and 15 with power reductions of $18 \%$ and $26 \%$ for DO and DD links. The fact that the benefits of antenna orientation as a parameter is retained at all densities shows that the size of the network has no bearing on the decision to have the topology construction algorithm derive an antenna orientation along with a power assignment. 


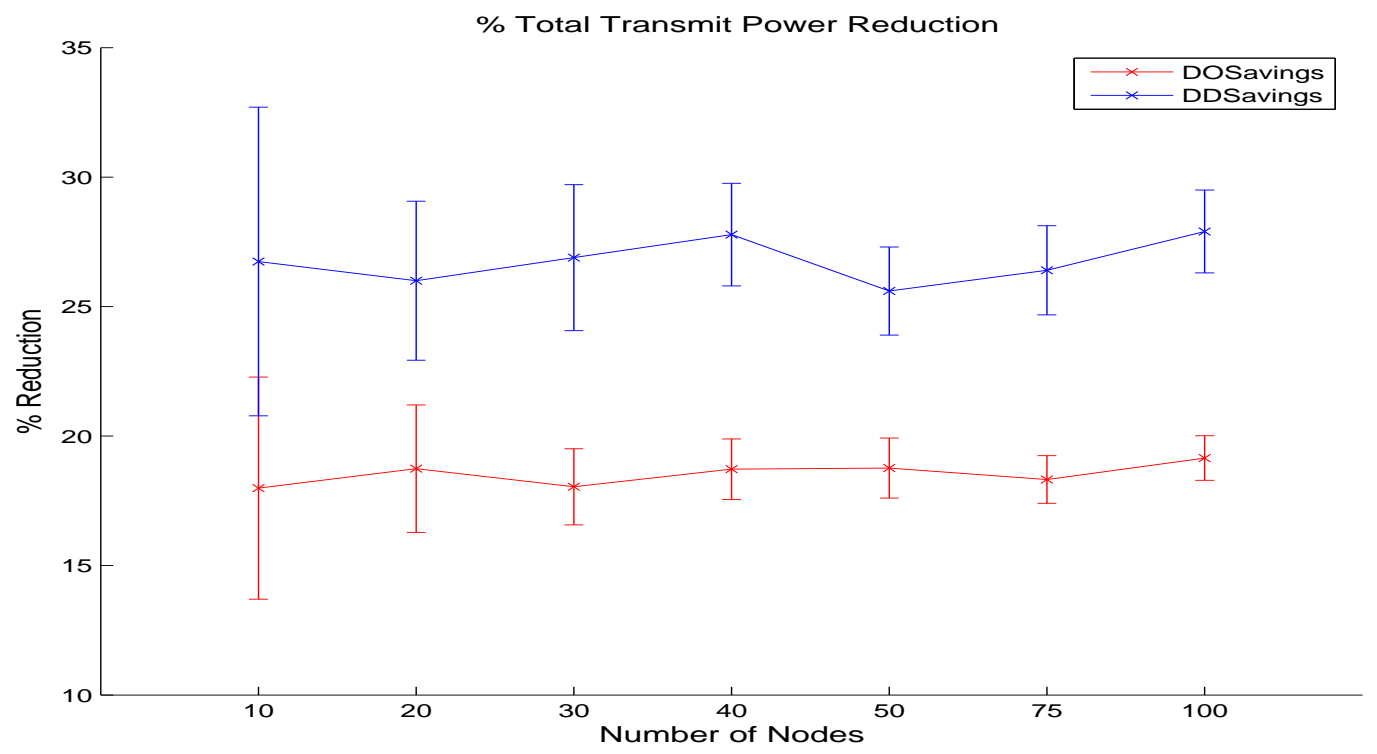

Fig. 15. Percentage savings by using antenna orientation as a parameter for TotalP metric

\subsection{Effect of varying beamwidth}

We assume that when the beamwidth is decreased or increased, the number of beams increases or decreases, maintaining a $360^{\circ}$ coverage around the node. Thus, increasing or decreasing the beamwidth does not have any positive or negative effects in terms of coverage. As Figures 16 and 17 show, the effect of increased beamwidth is to require higher powers which follows from the fact that the value of maximum gain decreases with increasing beamwidth. The benefits of using antenna orientation as a parameter are again evident regardless of the beamwidth.

\subsection{Accuracy of Heuristic Algorithms}

Here we compare the power consumption of our heuristic algorithms against their optimal counterparts. The optimal algorithms exhaustively search for an ideal antenna orientation and power assignment for a given set of nodes and their locations. Due to the intractable nature of computation involved, this experiment was run for networks with 10 nodes randomly deployed in a $25 \times 25$ square area with $\theta=15^{\circ}$. The beamwidth was kept small to increase the gains, the effect of which could possibly result in a larger difference between the optimal and heuristic algorithms.

As shown in Table 1, the heuristic algorithms are much more power efficient than their stated approximation bounds. The PADA-DD heuristic algorithms 


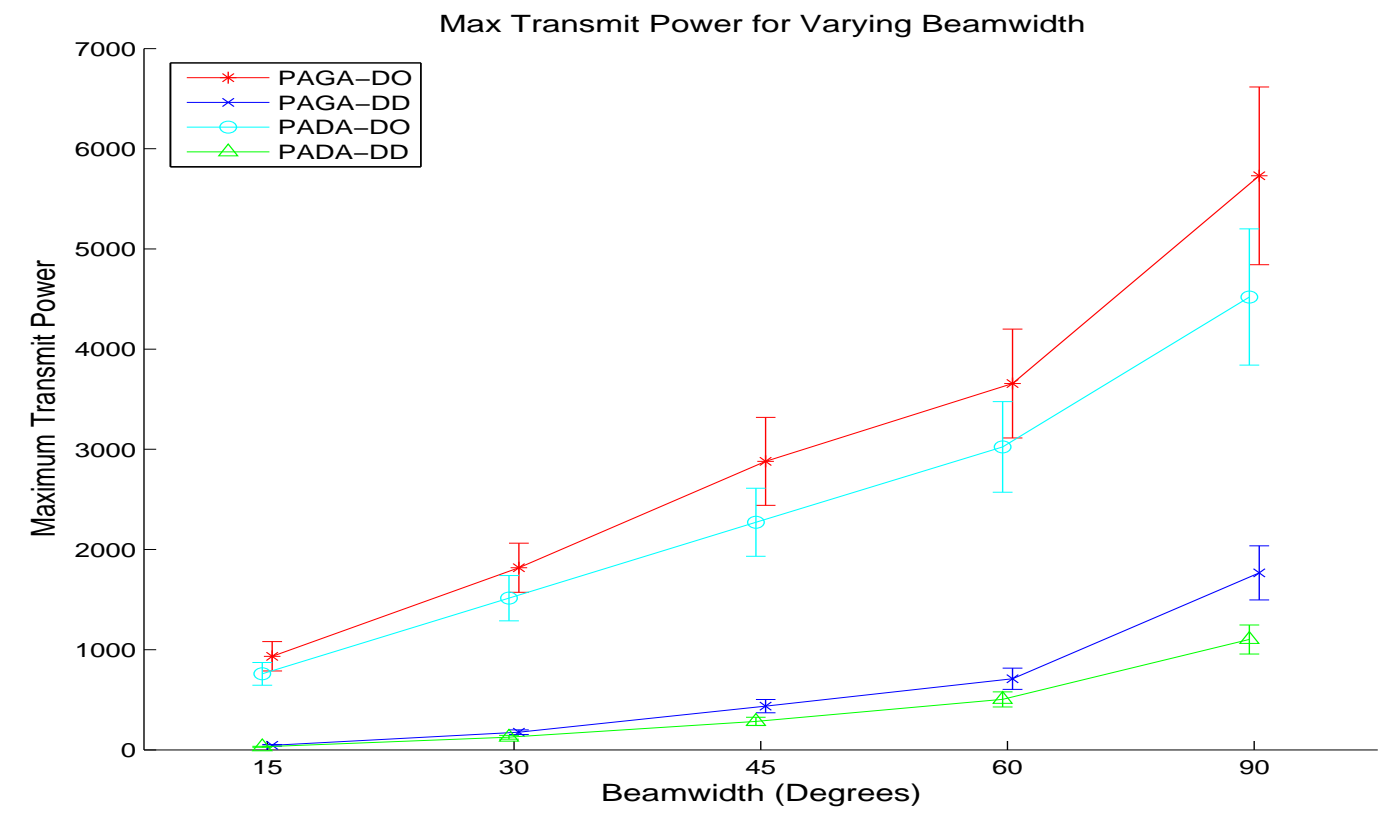

Fig. 16. Comparing the effect of beamwidth on maximum transmit power for all schemes for MaxP metric

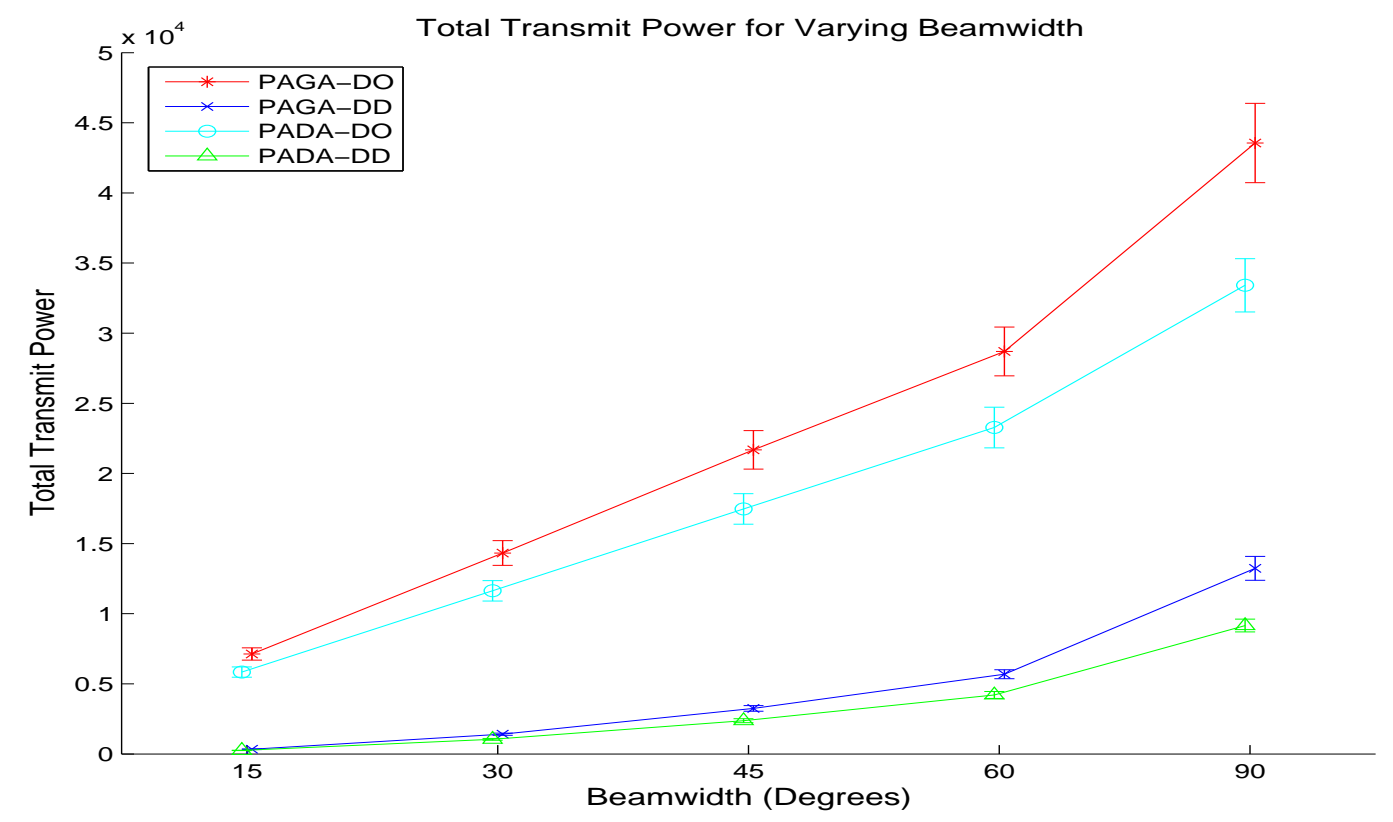

Fig. 17. Comparing the effect of beamwidth on maximum transmit power for all schemes for TotalP metric

turn out to be more accurate than PADA-DO ones, possibly due to the presence of symmetric links. In general, the accuracy of the algorithms is closer to the optimum for the MaxP metric than TotalP metric. This can be expected due to the cumulative nature of the latter metric. Thus, at least for small networks, it can be verified that our heuristics can be used for producing power efficient topologies with polynomial running time algorithms. For larger 
Table 1

Heuristic vs Optimal Values (\% Difference in Power)

\begin{tabular}{|l||l|l||l|l|}
\hline \multicolumn{1}{|c||}{} & \multicolumn{2}{l|}{ PADA-DO } & \multicolumn{2}{l|}{ PADA-DD } \\
\cline { 2 - 5 } & MaxP & TotalP & MaxP & TotalP \\
\hline \hline Average(\%) & 0.04 & 7.05 & 0.05 & 2.51 \\
Standard Deviation(\%) & 0.38 & 6.60 & 0.32 & 3.53 \\
Maximum(\%) & 4.83 & 32.29 & 2.43 & 18.00 \\
\hline
\end{tabular}

networks, we need to rely on the approximation bounds derived for guarantees in power savings.

\section{Conclusion}

A case was made for modeling switched beam directional antennas with a non-uniform model of gain within the beamwidth. Algorithms were presented for power-efficient topology construction using this non-uniform model of gain with bounds on their accuracy. It was shown through evaluation that using antenna orientation as a parameter in the topology construction algorithm leads to significant reduction in maximum as well as total power of the network.

\section{Acknowledgment}

This work is supported in part by NSF grants CCF-329794 and ANI-0208116.

\section{References}

[1] V. Namboodiri, L. Gao, R. Janaswamy, Power-efficient topology control for wireless networks with switched beam directional antennas, in Proceedings of IEEE MASS, 2005.

[2] E. Althaus, G. Calinescu, I. Mandoiu, S.Prasad, N. Tchervenski, and A. Zelikovsly, Power efficient range assignment for symmetric connectivity in static ad hoc wireless networks, Wireless Networks, accepted for publication.

[3] A.E.F. Clementi, P. Penna, and R. Silvestri, On the power assignment problem in radio networks, Electronic Colloquium on Computational Complexity (ECCC), (054), 2000. 
[4] L.M. Kirousis, E. Kranakis, D. Krizanc, and A. Pelc, Power consumption in packet radio networks, Theoretical Computer Science, 243:289-305, 2000.

[5] E.L. Lloyd , R. Liu , M.V. Marathe , R. Ramanathan , S. S. Ravi, Algorithmic aspects of topology control problems for ad hoc networks, in Proceedings of ACM MOBIHOC, 2002.

[6] R. Ramanathan and R. Rosales-Hain, Topology control of multihop wireless networks using transmit power adjustment, in Proceedings of IEEE INFOCOM, 2000 .

[7] P. Santi, Topology control in wireless ad hoc and sensor networks, Wiley, 2005.

[8] S. Guo, O.W. Yang, Antenna orientation optimization for minimum-energy multicast tree construction in wireless ad hoc networks with directional antennas, in Proceedings of ACM MOBIHOC, 2004.

[9] Z. Huang, C. Shen, C. Srisathapornphat, C. Jaikaeo, Topology control for ad hoc networks with directional antennas, in Proceedings of IEEE ICCCN, 2002.

[10] I. Kang and R. Poovendran, S-GPBE: A power-efficient broadcast routing algorithm using sectored antenna, in Proceedings of IASTED WOC, 2003.

[11] A. Spyropoulos, and C. S. Raghavendra, Energy efficient communication in ad hoc networks using directional antennas, in Proceedings of IEEE INFOCOM, 2002 .

[12] J. E. Wieselthier, G. D. Nguyen, and A. Ephremides, Energy limited wireless networking with directional antennas: The case of session-based multicasting, in Proceedings of IEEE INFOCOM, 2002.

[13] A. Spyropoulos, C. S. Raghavendra, Capacity bounds for ad-hoc networks using directional antennas, in Proceedings of ICC, 2003.

[14] S. Yi , Y. Pei , S. Kalyanaraman, On the capacity improvement of ad hoc wireless networks using directional antennas, in Proceedings of ACM MOBIHOC, 2003.

[15] R. Ramanathan, On the performance of ad hoc networks with beamforming antennas, in Proceedings of ACM MOBIHOC, 2001.

[16] T. Rappaport, Wireless communications: Principles and practice, Prentice Hall, 2002.

[17] M. Takai, J. Zhou and R. Bagrodia, Adaptive range control using directional antennas in mobile ad hoc networks, in Proceedings of MSWIM, 2003.

[18] R. Janaswamy, D. Gupta, K. Wood, and A. Mathews, Feasibility of $60 \mathrm{GHz}$ for high speed indoor wireless LANs, IEEE Trans. Commun., submitted for publication.

[19] M. Marina and S. Das, Routing performance in the presence of unidirectional links in multihop wireless networks, in Proceedings of ACM MOBIHOC, 2002. 
[20] R Prim, Shortest connection networks and some generalizations, Bell System Technical Journal 1957, 36:1389-1401.

[21] R.G. Gallager, P. A. Humblet, and P. M. Spira, A distributed algorithm for minimum-weight spanning trees, ACM TOPLAS, vol.5, no. 1, pp. 66-77, January 1983.

[22] N. Li, C.-J. Hou, and L. Sha, Design and analysis of an MST-based topology control algorithm, in Proceedings of IEEE INFOCOM, 2003. 